\title{
Vulnerabilidad sísmica y capacidad de carga de un puente en acero basado en confiabilidad estructural
}

\section{Seismic vulnerability and load carrying capacity studies of a steel bridge based on structural reliability}

\author{
Edgar Muñoz*1, Federico Núñez, Jorge A. Rodríguez, Alfonso Ramos, Camilo O tálora
}

* Pontificia U niversidad Javeriana. CO LO M BIA

Resumen

Fecha de recepción: 29/10/2008 Fecha de aceptación: 28/ 11/ 2008 PAG. $125-144$

Por medio de la confiabilidad estructural, para un Puente en Colombia después de su monitoreo. Los resultados de este trabajo son parte de una nueva metodología para evaluar puentes existentes mediante una colaboración entre Universidad-Empresas-Gobierno Nacional. Este estudio ayuda en la toma de decisiones y en la priorización de tareas de rehabilitación. Este estudio incluyó: caracterización dinámica de las vibraciones naturales del puente; adquisición de datos de fuerzas internas debidas al tráfico por medio de tecnología de LVDT's y Strain Gages; evaluación de las fuerzas actuales comparadas contra los esfuerzos máximos permitidos; modelación estática, dinámica y estructural. Adicionalmente se hizo un estudio de amenaza sísmica y respuesta dinámica del área del puente y nueve (9) diferentes espectros de respuesta fueron obtenidos, cada uno con un periodo de retorno diferente. Se encontró que las torres del puente tienen una probabilidad de falla mayor que la permitida por los códigos. Lo anterior condujo a un reforzamiento inmediato del puente.

Palabras Clave: Confiabilidad estructural, puentes, modelo estructural, vulnerabilidad sísmica, sobrecarga, efectos locales, instrumentación, probabilidad de falla, índice de confiabilidad

Abstract

In this document, seismic vulnerability and load carrying capacity analyses are presented for a bridge in Colombia after monitoring by means of reliability of structures. Results of this work are part of a new methodology to evaluate existing bridges carried out though a partnership among University - Private Companies - and Goverment. This study helps in decision making on the priority of rehabilitation tasks. This study included: dynamic characterization of natural vibrations of the bridge; data logging of inner forces due to traffic by using LVDT's and strain gages technology; evaluation of actual forces to be compared to allowed stresses, dynamic and static structural modelling. Also, a seismic hazard and dynamic response of the local area of the bridge was developed and nine (9) different response spectra were obtained, each one varying its return period. It was found that the bridge towers have a larger failure probability than that allowed by the Codes. This produced an inmediate strengthening of the bridge.

Keywords: Structural reliability, bridges, structural model, seismic vulnerability, overloads, local effects, instrumentation, failure probability, reliability index

\section{Introducción}

Desde hace ocho años el grupo de investigación de "Estructuras" del Departamento de Ingeniería Civil de la Pontificia Universidad Javeriana, viene trabajando en el tema de evaluación de puente vehiculares existentes mediante técnicas de confiabilidad estructural, teniendo en cuenta algunos de los avances en el estado del conocimiento internacional (Sobrino et al., 1993; Tanner et al., 1998a; Tanner et al., 1998b). Este Grupo, reconocido por CO LCIEN CIAS (Instituto Colombiano para la Ciencia

\footnotetext{
1 Autor de correspondencia / Corresponding author:

Grupo Estructuras. Facultad de Ingenieria, Pontificia U niversidad Javeriana, Colombia. E-mail: edgar.munoz@javeriana.edu.co
}

y la Tecnología), ha realizado estudios de confiabilidad estructural de los siguientes puentes del País (adicionalmente al puente que se presente en este documento): Puente Puerto Salgar, Viaducto atirantado Dos Q uebradas y Puente Río N egro. Estos estudios han sido un primer aporte para la ingeniería de puentes, que son semilla o base para el desarrollo e implementación de una norma Colombiana para la revisión de puentes existentes. En ellos se ha encontrado las ventajas de la confiabilidad estructural y la importancia de hacer la revisión del camión de carga viva para la evaluación de puentes existentes (Muñoz, et al., 2002; Muñoz y 
Valbuena, 2005; Muñoz et al, 2006).

También realizó un estudio sobre las causas del colapso total o parcial de algunos de los puentes en Colombia, con el cual se identificaron sus vulnerabilidades principales, encontrándose que una de ellas son las deficiencias estructurales (M uñoz, 2002).

De esta forma se han hecho los primeros aportes tecnológicos e innovadores en este tema a la Ingeniería $\mathrm{N}$ acional, trabajando en una alianza estratégica: Academia - Estado - Empresas de Ingeniería. Es así como este equipo, con apoyo del INVIAS (Instituto Nacional de Vías, Colombia) y trabajando conjuntamente con los Contratistas, realizó el estudio de vulnerabilidad del puente Cajamarca, en la carretera La Línea-Ibagué (Departamento del Tolima, Colombia) a través de técnicas de confiabilidad estructural, instrumentación y monitoreo. Dicho puente fue construido por la firma Alemana "Fried Group PK", entre los años de 1957 a 1959. Tiene una superestructura continua de tres luces con una longitud total de 283 metros. Compuesto por dos armaduras de acero de paso inferior y un tablero de sección mixta con losa en concreto reforzado con un ancho total de 9,68 metros (Figura 1 y Figura 2), juntas de dilatación, dos andenes y barandas en acero. La losa en concreto reforzado se apoya en cuatro vigas de acero longitudinales, las cuales transmiten su carga a vigas transversales de acero y estas se apoyan en los nudos del cordón superior de las armaduras de acero de paso inferior.

Los elementos de las armaduras constan de conexiones remachadas y se unen a través de arriostramientos en la parte superior, inferior y en sentido transversal. El puente consta de dos torres de acero en celosía de 71 metros de altura cimentados en cajones en concreto reforzado. Además dos estribos en concreto reforzado, de los cuales no se conoce su tipo de cimentación, ya que no se encontraron planos ni memorias de cálculo, ni estudios técnicos que lo especifiquen. En 1996 el INVIAS contrató obras de mantenimiento y rehabilitación por lo que la estructura en acero del puente tiene refuerzos de actualización (platinas soldadas) en cordones inferiores y superiores de las armaduras.

Para el estudio se contó con la siguiente información técnica: un estudio de consultoría de diagnóstico y seguimiento para al rehabilitación de 1996, un informe de inspección del puente metálico de Cajamarca en el Tolima, planos estructurales del puente e informes de resultados de ensayos a compresión y modulo de elasticidad de la losa en concreto realizados por diferentes empresas.

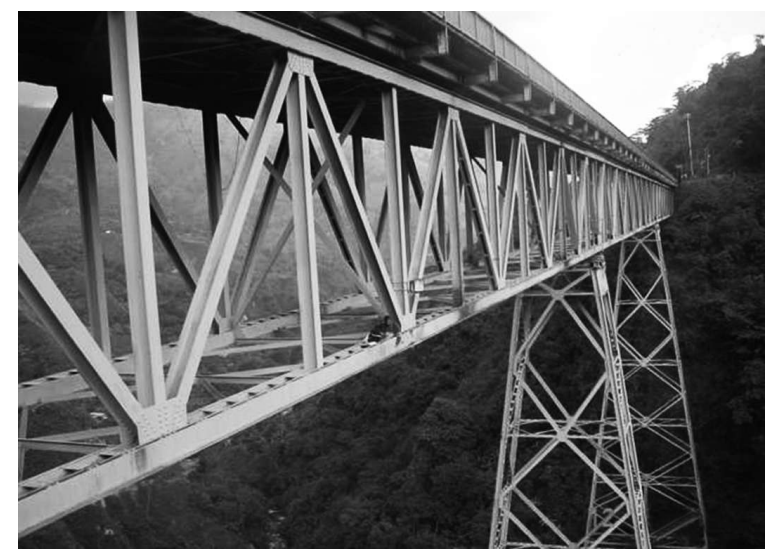

Figura 1. Armadura de paso inferior y dos torres de celosía en acero

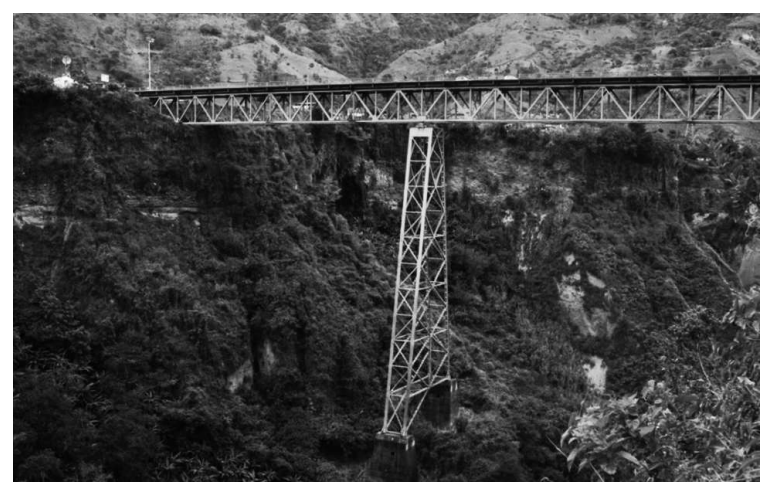

Figura 2. Vista en perfil del puente y torre del lado de Cajamarca

\section{Desarrollo del Trabajo}

En la Figura 3, se presentan un organigrama con las etapas llevadas a acabo para el desarrollo de este estudio. Cada una de estas etapas se explica a continuación.

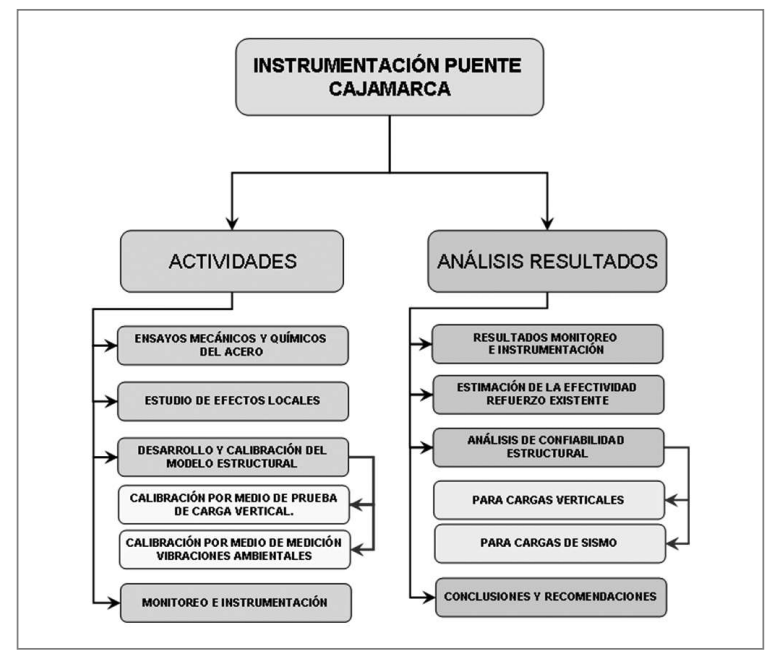

Figura 3. Etapas del estudio realizado sobre el puente Cajamarca 


\subsection{Ensayos mecánicos y químicos del acero}

En laboratorio de materiales de la U niversidad se realizaron ensayos de tensión a 8 muestras del puente (Figura 4), extraídas de elementos no principales de su estructura. Los resultados de los ensayos de tensión realizados a las ocho muestras de acero se presentan en la Figura 5. Se encontró que este acero es tipo A-33. Los ensayos químicos y de metalurgia realizados a la muestra de acero concluyeron que presenta inclusiones tipo A, serie fina y tipo D3 serie gruesa. Presenta una microestructura compuesta por ferrita y un tamaño de grano aproximado ASTM 6.

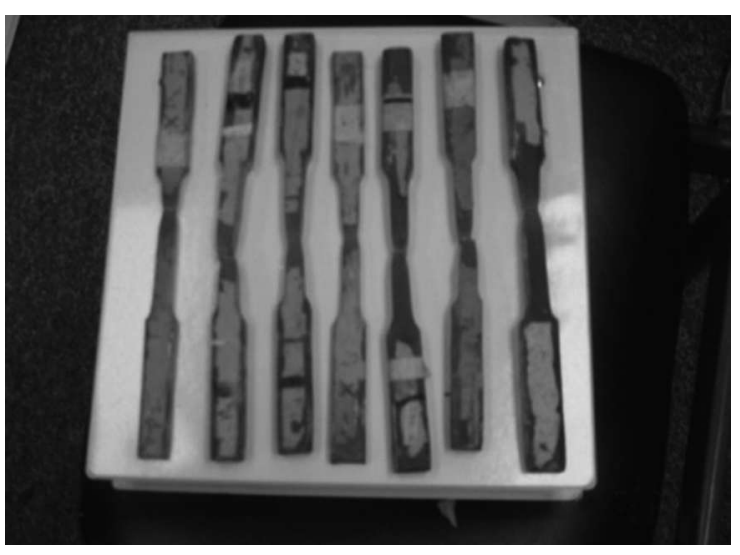

Figura 4. Siete de las ocho muestras de acero ensayadas en el laboratorio de la U niversidad

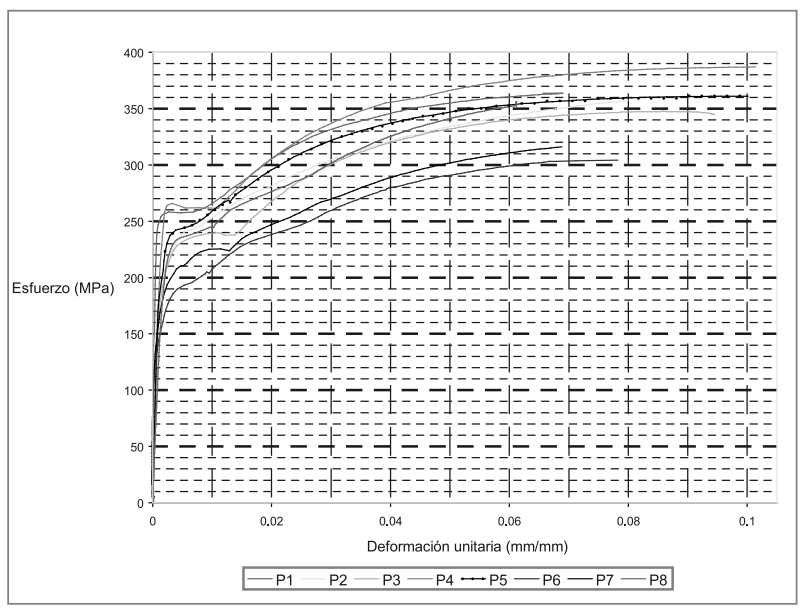

Figura 5. Diagramas de esfuerzo deformación de las ocho probetas del puente

\subsection{Estudio de efectos locales}

Para el estudio de respuesta sísmica local se realizó una exploración geofísica con refracción sísmica del suelo donde esta cimentado este puente teniendo en cuenta la profundidad y dureza de la roca, contacto entre capas; continuidad del modelo interpretativo, velocidades compresionales; velocidades de corte; módulos del subsuelo a pequeñas deformaciones, módulos elásticos del subsuelo conocidos como módulo de Young $(E)$, módulo de corte máximo (Go), módulo de deformación volumétrica $(K)$, relación de Poisson y correlaciones entre las velocidades halladas y la geología local. La exploración incluyó la realización de $385 \mathrm{~m}$ de líneas de refracción sísmica distribuidas en 7 líneas de 55m de longitud, algunas de ellas individuales y otras unidas formando perfiles, con el fin de conocer las características geotécnicas, sísmicas y módulos elásticos de los materiales.

Se realizó una evaluación de la amenaza sísmica y respuesta dinámica del sitio del Puente de Cajamarca, la cual permite identificar los efectos de los suelos y la topografía, que en este caso son muy importantes debido a los contrastes de materiales y la fuerte expresión topográfica. Para la realización de este componente del estudio se investigó la amenaza sísmica esperada para el sitio con el fin de identificar registros de sismos representativos para los análisis de respuesta. La primera parte, consintió en la evaluación de espectros de amenaza sísmica uniforme de aceleraciones en roca. Esto incluyó un análisis de confiabilidad, para lo cual fue necesario contar con valores probabilísticos de la amenaza sísmica.

El modelo sismológico utilizado en este trabajo es extractado del Estudio General de Amenaza Sísmica de Colombia (IN GEO MINAS-UN IANDES, 1996). Para realizar el análisis de amenaza se examinó como área de influencia una zona circular de $200 \mathrm{~km}$ de radio centrada en el puente de Cajamarca cuyas coordenadas geográficas son: $75.42 \mathrm{~W}$ y $4.44 \mathrm{~N}$. Dentro del área de influencia existen 10 fuentes sismogénicas, 9 de ellas se simulan como líneas fuentes y 1 se representa como un área circular con igual probabilidad de generación de eventos denominada "Ninguna fuente". Las fuentes identificadas en el estudio son: Benioff intermedia, Benioff profunda , Cauca, Frontal, Garrapatas, Ibagué, M urindóAtrato, Palestina, Romeral, Salinas y Ninguna Fuente. Mediante un programa de generación de espectros, desarrollado por el ingeniero Jorge Alonso Prieto, se determinaron nueve espectros de respuesta diferentes. Este programa se basó en la variación de algunos parámetros de la ecuación de atenuación definida por la geología, las distancias a las principales fallas geológicas de la región y los datos obtenidos para los eventos sísmicos registrados en los diferentes medidores localizados cercanos a la zona. El programa se utilizó introduciendo la ecuación de atenuación (Ambrasseys et al., 2000), definiendo la aceleración absoluta para 
diferentes periodos espectrales que varían desde cero hasta 2 segundos. Además tiene en cuenta la frecuencia anual de excedencia para el evento sísmico definido por ese espectro de respuesta (i.e. el inverso matemático del periodo de retorno para ese evento sísmico definido por ese espectro de respuesta). Resuelve evalúa la integral dada en la ecuación (1), numéricamente. Para el presente caso, la función de densidad de amenaza sísmica se consideró dependiente de la aceleración espectral sa(T), la distancia al sitio, r y la magnitud, $m$. Es decir la función de densidad es $\mathrm{f}(\mathrm{sa}, \mathrm{m}, \mathrm{r})$. Por tanto y similarmente al caso unidimensional, la probabilidad de que se exceda un valor de aceleración espectral en la fuente sísmica i es:

$$
P(S A \geq s a)_{i}=\int_{a}^{s a \max } \int_{n o}^{m u} \int_{o}^{\max } f(s a, r, m) d r d m d s a
$$

Los intervalos de evaluación de las funciones dadas en la mencionada integral fueron: para los radios, los correspondientes a intervalos de longitud medidos en la falla menor a $10 \mathrm{~km}$. Para las magnitudes intervalos de 0.5 unidades de magnitud. El programa fija un valor de aceleración espectral y calcula la probabilidad y el periodo de retorno correspondiente. Por tanto para encontrar las ordenadas espectrales para diferente periodos de retorno se deben realizar iteraciones. Los datos para el espectro de amenaza uniforme en la roca (aceleración en gravedad y periodo en segundos) para diferentes periodos de retorno y el $5 \%$ de amortiguamiento con respecto al crítico se presentan en la Figura 6.

Para la determinación de los espectros de respuesta en superficie, se consideraron los eventos sísmicos que se han identificado como representativos para la amenaza sísmica del sitio considerando los eventos correspondientes al sismo de diseño de acuerdo con la NSR 98. Se identificaron las fuentes sísmicas representativas así como los posibles sismos en términos de magnitud y distancia que se pueden esperar para el sitio como sismos de diseño. Con base en este análisis se identificaron una serie de registros que se utilizaron para los análisis de respuesta. Para el análisis de respuesta sísmica de las márgenes del Río Anaime, donde están apoyadas las pilas del puente, se hicieron análisis de propagación bidimensional de ondas con el prodîrma PLAXIS Profesional V.8.0. Para análisis dinámicos el programa utiliza un esquema implícito incondicionalmente convergente de $\mathrm{N}$ ewmark de integración paso a paso en el tiempo. El amortiguamiento se calcula con base en la frecuencia predominante del sismo, el rango de frecuencias de interés para los análisis y el amortiguamiento asumido de los materiales para calcular los parámetros de amortiguamiento de Rayleigh que utiliza el modelo. El programa tiene incorporadas fronteras absorbentes para evitar que las ondas que se propagan en el modelo se reflejen en las fronteras laterales. El sismo se aplica en la base del modelo (Figura 7).

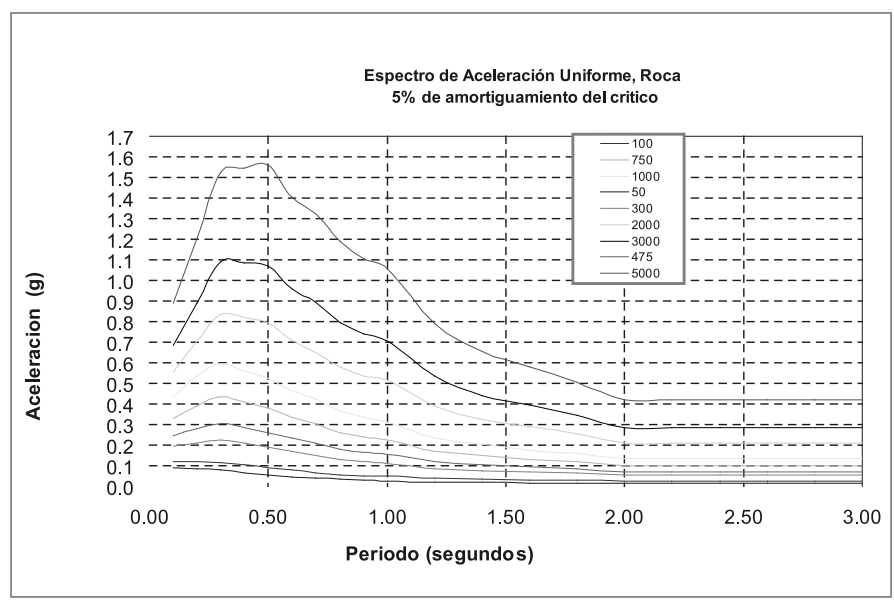

Figura 6. Espectros de respuesta para diferentes periodos de retorno

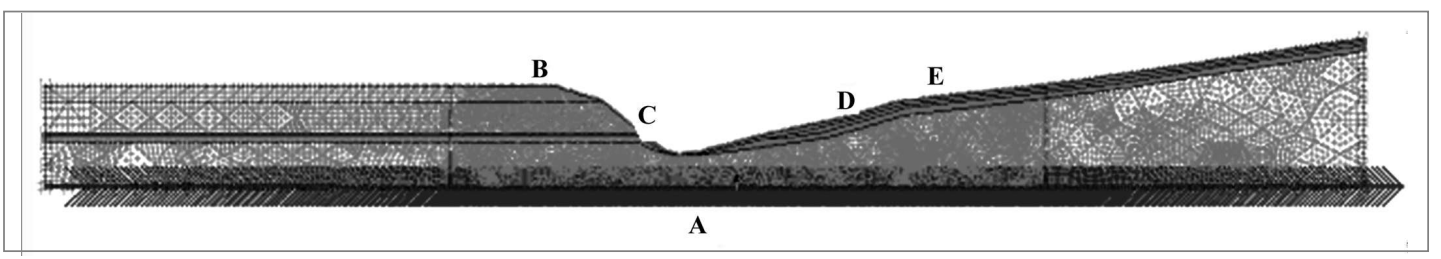

Figura 7. Sección de análisis - sección transversal. Modelo en elementos finitos 
Para estos modelos se obtuvo información topográfica de planos del IGAC y se identificaron las propiedades dinámicas de los materiales del área con base en los resultados de ensayos de refracción sísmica. Para efectos de utilizar los espectros de amenaza uniforme para los análisis de confiabilidad se obtuvieron funciones de amplificación para cada uno de los puntos de apoyo del puente y estas se aplicaron a los espectros de amenaza uniforme calculados con métodos probabilísticas como ya se indicó.

Con base en los resultados de la definición del espectro de diseño en roca para el sitio a partir de análisis pseudo-determinísticos y los resultados de los análisis probabilísticos presentados por la AIS (1996), se procedió a determinar seis sismos con características compatibles con los datos de la amenaza sísmica para el sitio. Los análisis representan el efecto del sismo sin tener en cuenta la interacción con la estructura ya que sólo se evaluó la respuesta en los puntos de apoyo del puente sin modelar el puente; sin embargo, en este caso el principal efecto en la amplificación de la señal es debido al cambio de materiales y a la geometría del cañón de la quebrada. Del modelo se obtuvieron las historias de aceleración en diferentes puntos en superficie donde se encuentran los sitios de apoyo de las pilas del puente y un punto en base de roca. La localización de los puntos de análisis se presenta en la Figura 7. Los resultados de los espectros de aceleraciones en los puntos evaluados se analizaron para obtener los espectros de respuesta para un 5\% de amortiguamiento, utilizando el programa DEGTRA 2000 (O rdaz et al., 2002) (UNAM - U niversidad Nacional Autónoma de México). En general, se observa que la diferencia en la respuesta sísmica que se presenta en las dos márgenes del Río es consecuencia fundamental de las características de los materiales de estas dos laderas.

Se observa que la amplificación de la respuesta en la margen del lado de Cajamarca con respecto a la de Ibagué es sustancialmente diferente y mayor debido a la presencia de los niveles aterrazados de Cajamarca (Q pt), los depósitos aluviales y materiales de flujos torrenciales y piroclásticos que son menos rígidos que los esquistos presentes en la margen de lado de Ibagué. En las Figura 8 y Figura 9 se presentan los espectros de aceleración para la torre de Ibagué y Cajamarca con varios valores de periodos de retorno. En la Figura 10 y Figura 11, se presenta una comparación entre los espectros de amenaza sísmica uniforme y los espectros de diseño determinados de acuerdo con el Código Colombiano de Diseño Sísmico de puentes (CCDSP).

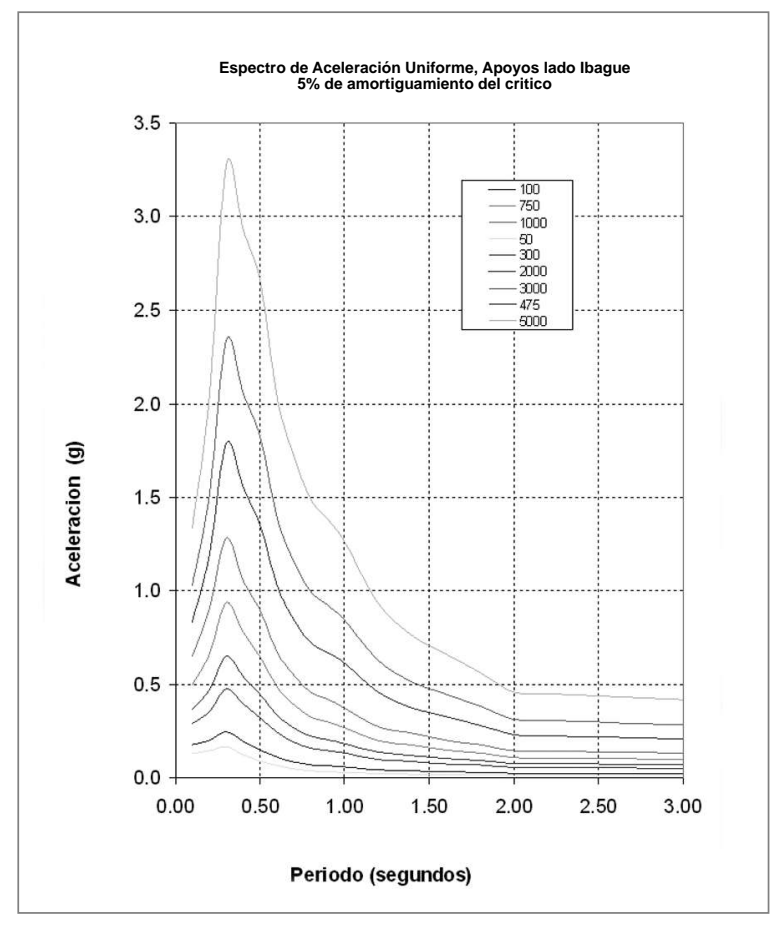

Figura 8. Apoyo Ibagué, $5 \%$ de amortiguamiento del crítico

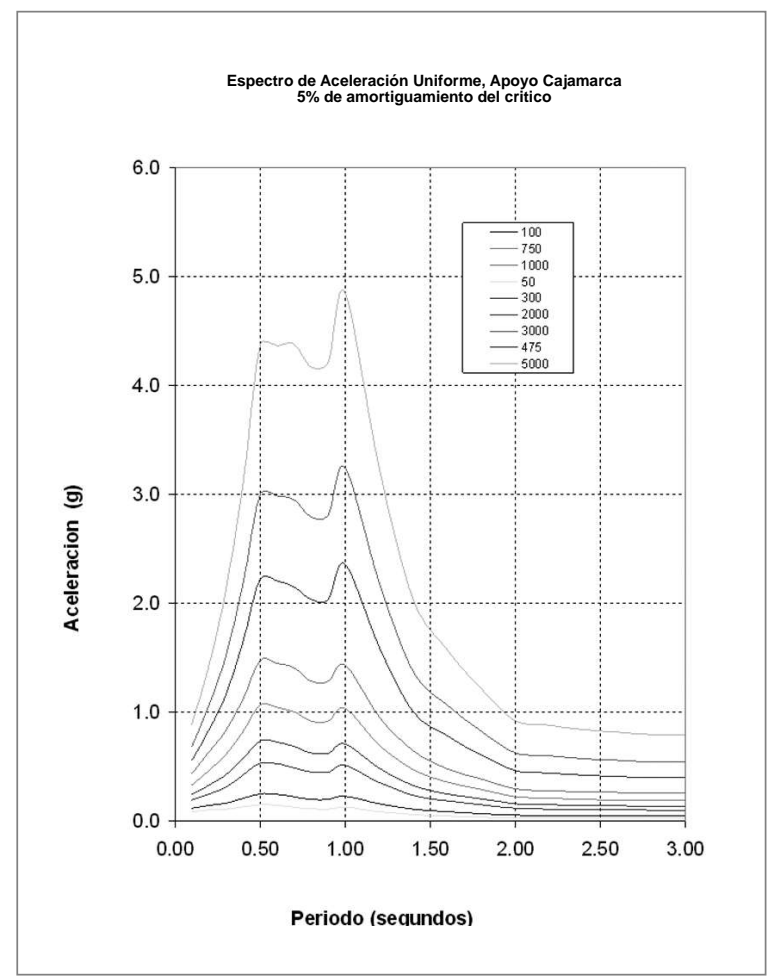

Figura 9. Apoyo Cajamarca, 5\% de amortiguamiento del crítico 


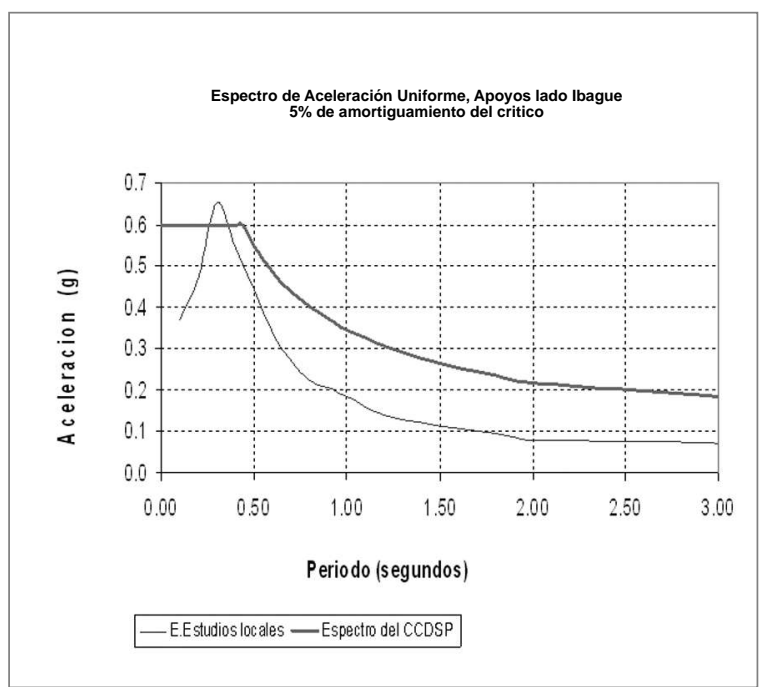

Figura 10. Comparación entre Espectro de Aceleración U niforme con $\mathrm{T}=475$ años y Espectro del CCDSP. Apoyo Ibagué

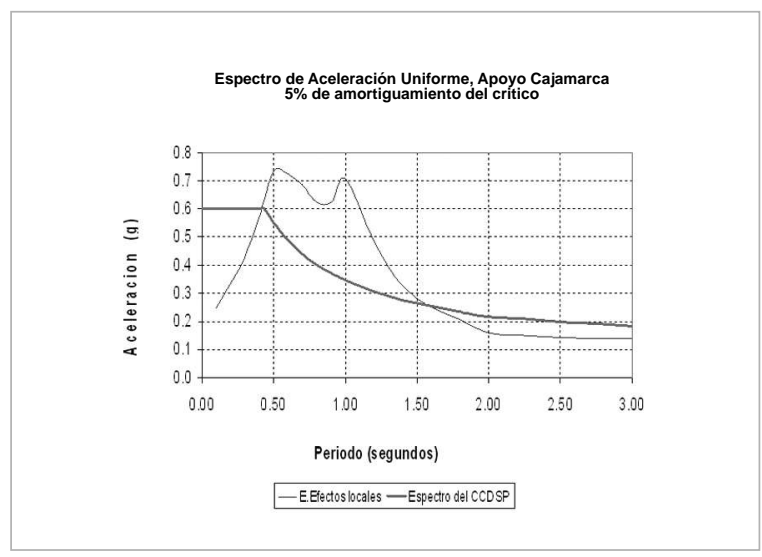

Figura 11. Comparación entre Espectro de Aceleración U niforme con $\mathrm{T}=475$ años y Espectro del CCDSP. Apoyo Cajamarca

\subsection{Desarrollo y calibración del modelo estructural}

Se desarrolló el modelo estructural del puente un en el programa SAP-2000 (Figura12), con el cual se realizó el análisis de confiabilidad, empleando planos "As Built" y un levantamiento estructural realizado por la Universidad y el Contratista.

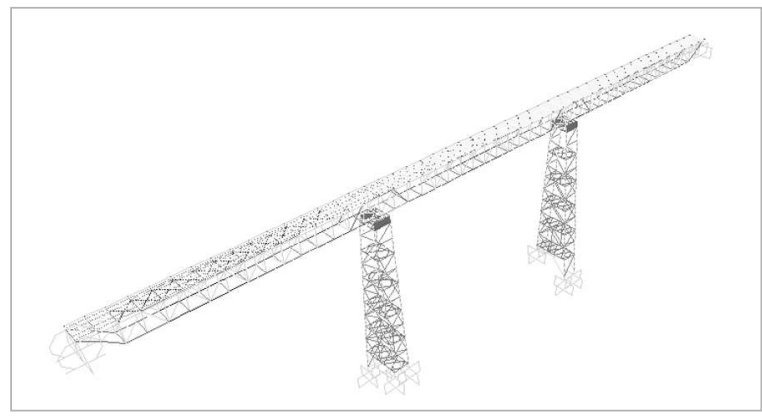

Figura 12. M odelo estructural del puente Cajamarca, desarrollado en SAP-2000
En este modelo los elementos de las torres y las armaduras se modelaron como elementos "Frame", cuyas secciones son armadas-compuestas y tienen forma de celosía. La losa en concreto reforzado se modeló mediante elementos finitos tipo "Shell". Este modelo también incluyó la rigidez que proporcionan las barandas y los andenes en cada lado del puente, lo cual se incluyó mediante elementos "Frame". También se incluyeron "Release" o liberación de grados de libertad en sus apoyos a elementos de arriostramientos cuyos apoyos en los extremos lo justifican (Conexiones a corte). El apoyo de la superestructura en el estribo de Ibagué se consideró articulado y el del estribo de Cajamarca se consideró libre en sentido longitudinal del puente (rodillos), con restricción en sentido vertical y transversal. Los apoyos de los montantes e consideraron articulados. El apoyo entre la superestructura y cada una de las torres se simuló en forma aproximada mediante un elemento "Frame", que tiene la geometría equivalente de las secciones de acero y un "Release" que pretende simular el comportamiento de los rodillos, donde se liberó el cortante y momento en sentido longitudinal del puente. Para su calibración se realizó una prueba de carga y la determinación de las propiedades dinámicas a través de las mediciones de vibraciones ambientales.

\subsubsection{Calibración por medio de prueba de carga}

Para la prueba de de carga se instalaron miras topográficas en cada una de las conexiones del cordón inferior de la armadura aguas arriba, que sirvieron para la toma de registros de las deformaciones en cada una de las hipótesis de la prueba de carga. Para las mediciones topográficas de todos los puntos se utilizaron tres. Se realizó dicha prueba mediante seis volquetas, debidamente cargadas, de las cuales se registró su geometría y peso por eje. Dicha prueba incluyó seis hipótesis de carga, consistente en dos en cada luz, una con las volquetas centradas y otra con las volquetas excéntricas. En la Figura 13, se presenta la comparación entre las deformaciones medidas para uno de las hipótesis en de prueba de carga (Seis volquetas centradas) con relación a las obtenidas del modelo estructural. En el proceso de calibración se decidió hacer modificaciones del modelo original, que se generaron modificando aspectos estructurales del modelo base que se explicó en el numeral anterior. En la Tabla 1 se presentan los porcentajes de error promedio entre la deformación máxima de los datos analíticos con respecto a los experimentales de los tres modelos estructurales. 


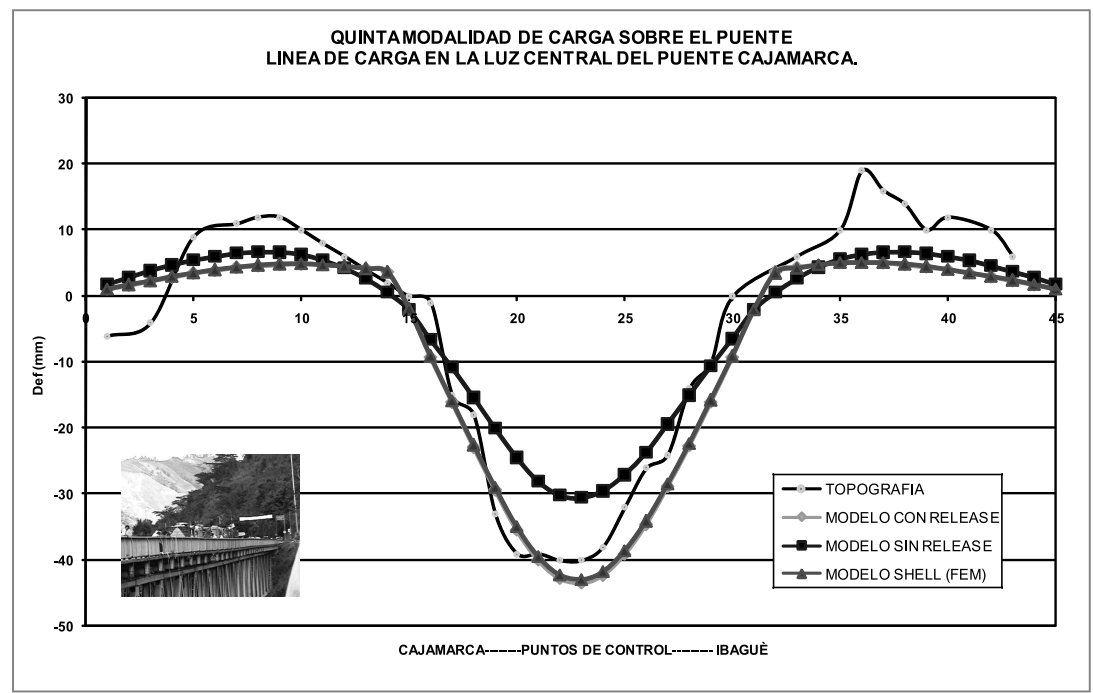

Figura 13. Comparación prueba de carga para la hipótesis 5

Tabla 1. Error al comparar los resultados experimentales contra los resultados analíticos de la prueba de carga

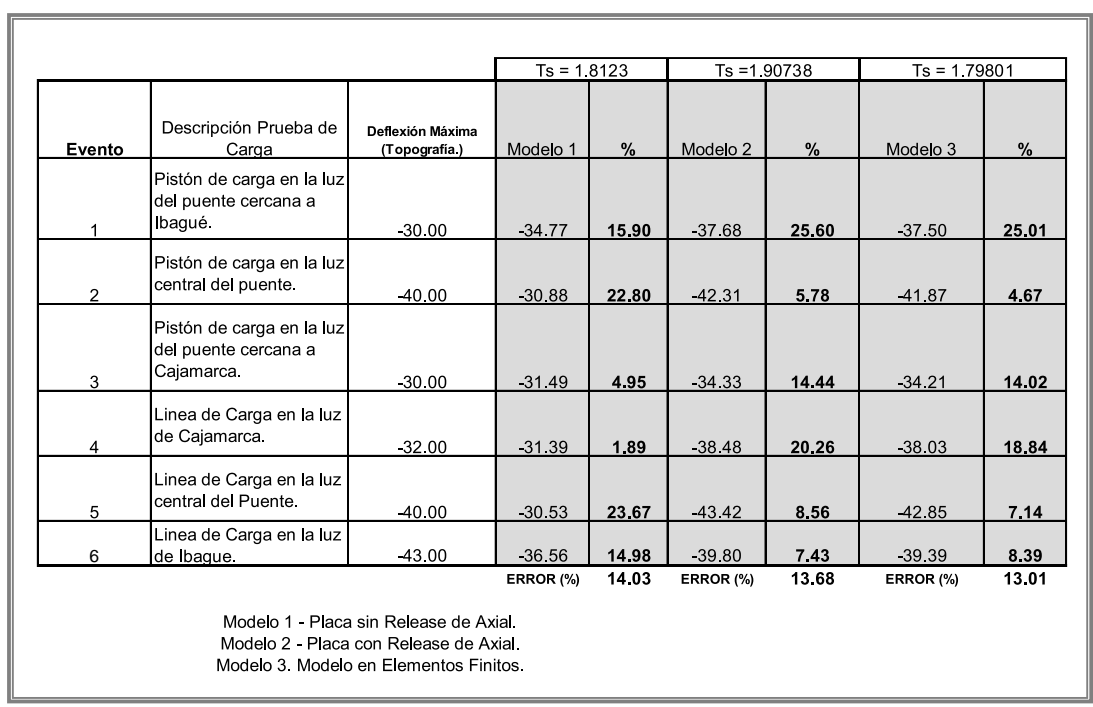

\subsubsection{Calibración con medición de vibraciones} ambientales

Para el estudio de la vulnerabilidad sísmica basada en confiabilidad estructural de este puente, es importante identificar experimentalmente sus propiedades dinámicas (frecuencias naturales, periodos predominantes y rigidez), para lo cual se realizaron mediciones de vibraciones ambientales. Para esto la estructura del puente se instrumentó mediante cuatro acelerómetros tipo Wilcoxon ${ }^{\circ} 731 \mathrm{~A}$, con amplificadores y tarjeta de adquisición de datos. Los sensores Wilcoxon $®$ toman 200 datos por segundos con un rango de frecuencia de interés entre 0 a $20 \mathrm{~Hz}$. Esta labor experimental se ejecutó cuando el puente estaba con tráfico y sin tráfico. En las Figura 15 y Figura 16 se presenta la ubicación de los acelerómetros en el puente, cuyas mediciones se hicieron en dos épocas diferentes.

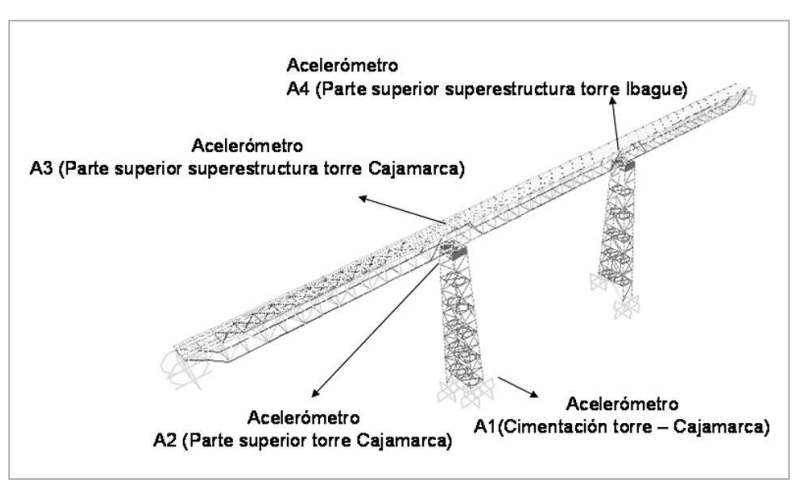

Figura 14. Localización de acelerómetros en las mediciones realizadas en el mes de diciembre de 2007 - Con tráfico 


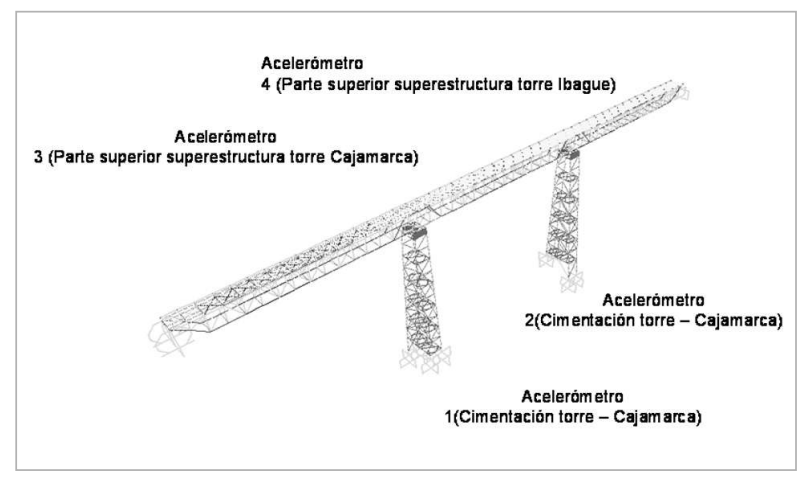

Figura 15. Localización de los acelerómetros en las mediciones realizadas en el mes de febrero de 2008 - Sin tráfico

A nivel nacional e internacional se han realizado estudios dinámicos sobre puentes, cuya experiencia ha sido importante y tenida en cuenta dentro del presente estudio (Gallego, 2007; Sarrazin, 2000; Binaria Ltda, 2006). Para la determinación de las propiedades dinámicas de este puente, se les realizó a cada una de las señales de los acelerómetros (acelerogramas), el siguiente procesamiento numérico, empleando un programa que se desarrolló en MatLabß).

- Corrección de línea base para los registros cuya línea base no se encuentra en cero

- Aplicación del filtro Pasabanda para eliminar ruido y ajustar las frecuencias del registro a un rango relacionadas con el tipo de estructura. En este caso se ajustaron dichos registros para una baja frecuencia de $0.1 \mathrm{~Hz}$ y una alta frecuencia de $20 \mathrm{~Hz}$

- Realizar análisis para pasar del dominio del tiempo al dominio de la frecuencia. Esto se logró a través de la transformada rápida de Fourier (FFT), la cual permite obtener el espectro de amplitud de Fourier para cada registro. Cada uno de estos espectros de amplitud se suavizó para eliminar el Aliasing relacionado con las altas frecuencias

- Se determinaron las funciones de transferencia de cada una de las pilas, para tener las vibraciones efectivas que tiene la estructura. Estas se realizaron al hacer un cociente entre la transformada de Fourier de las señales filtradas de la parte superior de la torre y la transformada de Fourier de las señales filtradas de su cimentación

Mediante este análisis de señales se determinaron las frecuencias naturales de la estructura del puente en el sentido transversal y longitudinal. Esto se revisó para las mediciones ejecutadas con tráfico en diciembre de 2007 y sin tráfico de febrero de 2008. Los resultados principales de este análisis se presentan en la Figura 17, Figura 18, Figura19 y Figura 20. Se encontró que en las mediciones de las vibraciones de la estructura con tráfico, estas se excitan, permitiendo identificar con mayor confiabilidad sus frecuencias predominantes (Tabla 2).

Los periodos y modos de vibración predominantes en el modelo estructural se aprecian en la Figura 20. Los errores entre las frecuencias experimentales con respecto a las analíticas se presentan en la Tabla 2.

\subsection{Monitoreo e instrumentación}

Para determinar la acción de las cargas de tráfico sobre algunos de los elementos principales del puente se emplearon equipos de instrumentación y monitoreo electrónicos. Estos consistieron en LVDT's de marca O MEGA (Transductor Variable Lineal de D esplazamiento). Se localizaron estos LVDT's en siete elementos principales del puente, empleando bases ancladas a la estructura metálica (Figura 21).

A través de este monitoreo se realizaron los registros de la fuerzas internas que se observan en la Figura 22, los cuales se compararon con los máximos permitidos producidos por la carga C-40-95 del Código Colombiano de Diseño Sísmico de Puentes (CCDSP). Mediante este estudio se comprobó que existe una sobrecarga importante que circula sobre el puente que afecta a uno de los montantes de la torre y a los cordones superior e inferior de la armadura. Esta sobrecarga coincide con los daños que tiene la soldadura que une las platinas de refuerzo con la sección original del cordón superior. También genera deterioro y daños a las conexiones y elementos principales del puente aumentando su riesgo y disminuyendo su seguridad.

\subsection{Estimación de la efectividad del refuerzo existente}

Con el objeto de verificar la efectividad de las platinas de refuerzo que se colocaron en el cordón inferior en el año de 1996 (O bras de rehabilitación), se instalaron LVDTs en la sección original y en dicha platina. Los registros de las fuerzas obtenidas en el monitoreo de las dos partes platina original (1956) y platina reciente(1996) se presentan en la Figura 23. Se encontró que la carga que toma la sección original es mucho mayor que la que toma la platina de refuerzo. La sección del elemento no funciona en forma solidaria debida posiblemente a las grietas encontradas en las soldaduras y los problemas de calidad de este refuerzo instalado en 1996 (Figura 24). 


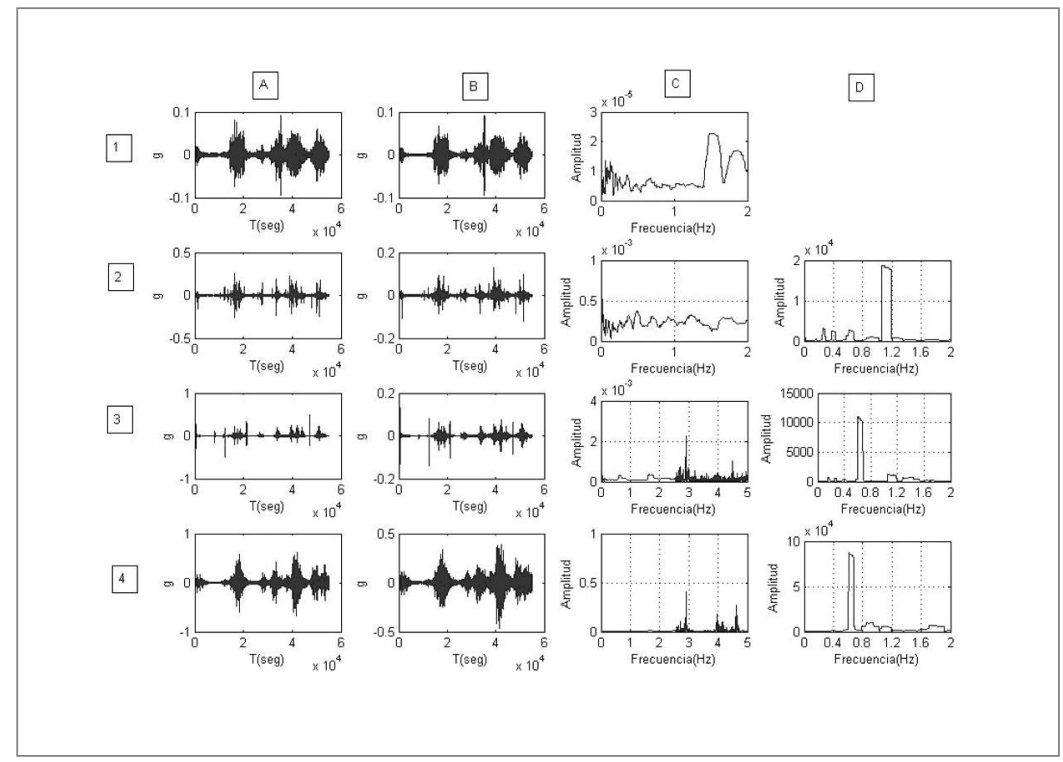

Figura 16. Resultados de vibraciones ambiéntales sentido transversal - Diciembre 2007 - Con tráfico ${ }^{1}$

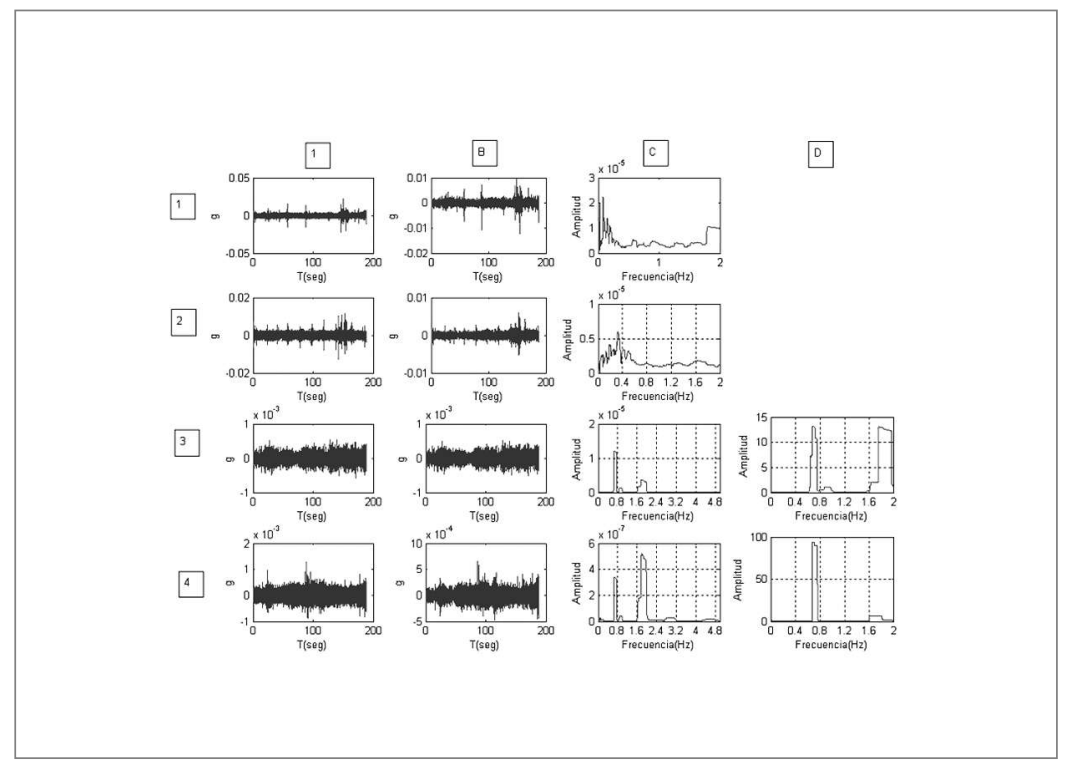

Figura 17. Resultados de vibraciones ambiéntales sentido transversal - Febrero 2008 - Sin tráfico 2

${ }^{1}$ (A1) Acelerograma 1 (sin filtrar). (B1) Acelerograma 1 (filtrado). (C1) Transformada de Fourier del registro del acelerómetro 1 (A2) Acelerograma 2 (sin filtrar). (B2) Acelerograma 2 (filtrado). (C2) Transformada de Fourier del registro del acelerómetro 2.(D2) Función de transferencia C2/C1. (A3) Acelerograma 3 (sin filtrar). (B3) Acelerograma 3 (filtrado). (C3) Transformada de Fourier del registro del acelerómetro

3.(D3) Función de transferencia C3/C1. (A4) Acelerograma 4 (sin filtrar). (B4) Acelerograma 4 (filtrado). (C4) Transformada de Fourier del registro del acelerómetro 4. (D 4) Función de transferencia C4/C1. 


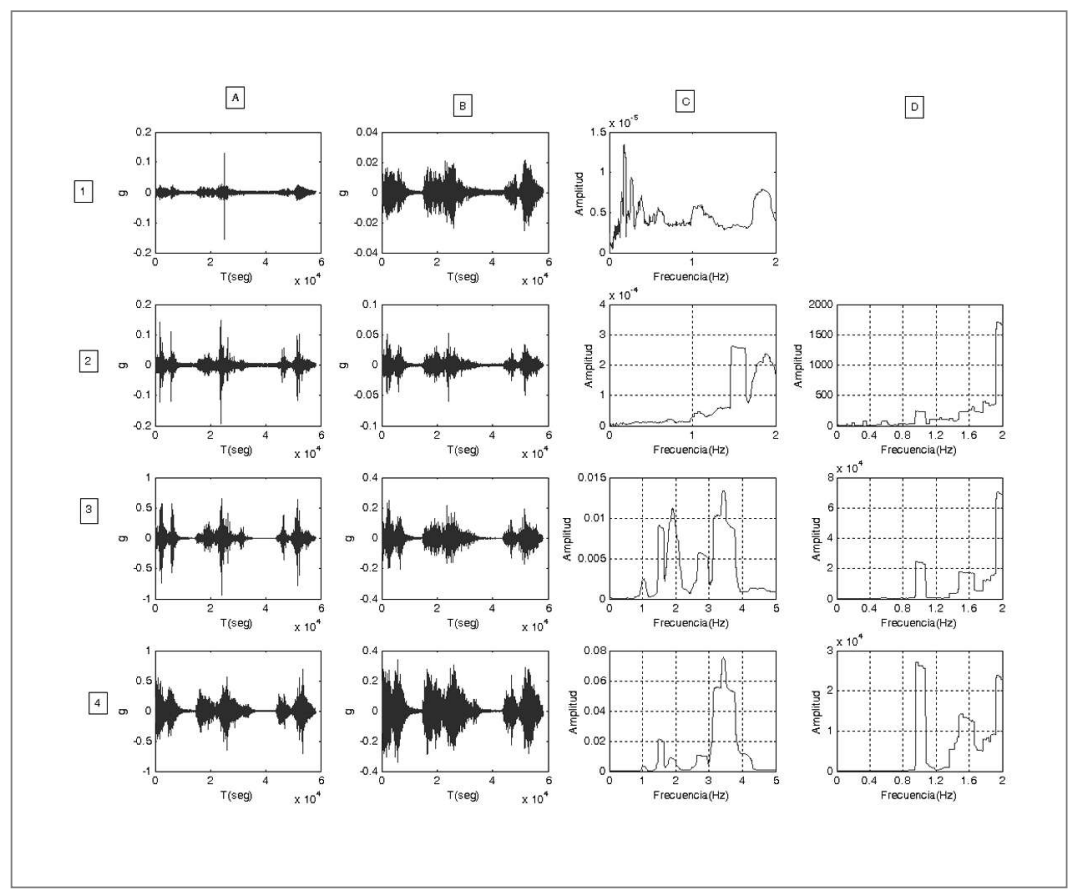

Figura 18. Resultados de vibraciones ambiéntales sentido longitudinal - Diciembre 2007 - Con tráfico²

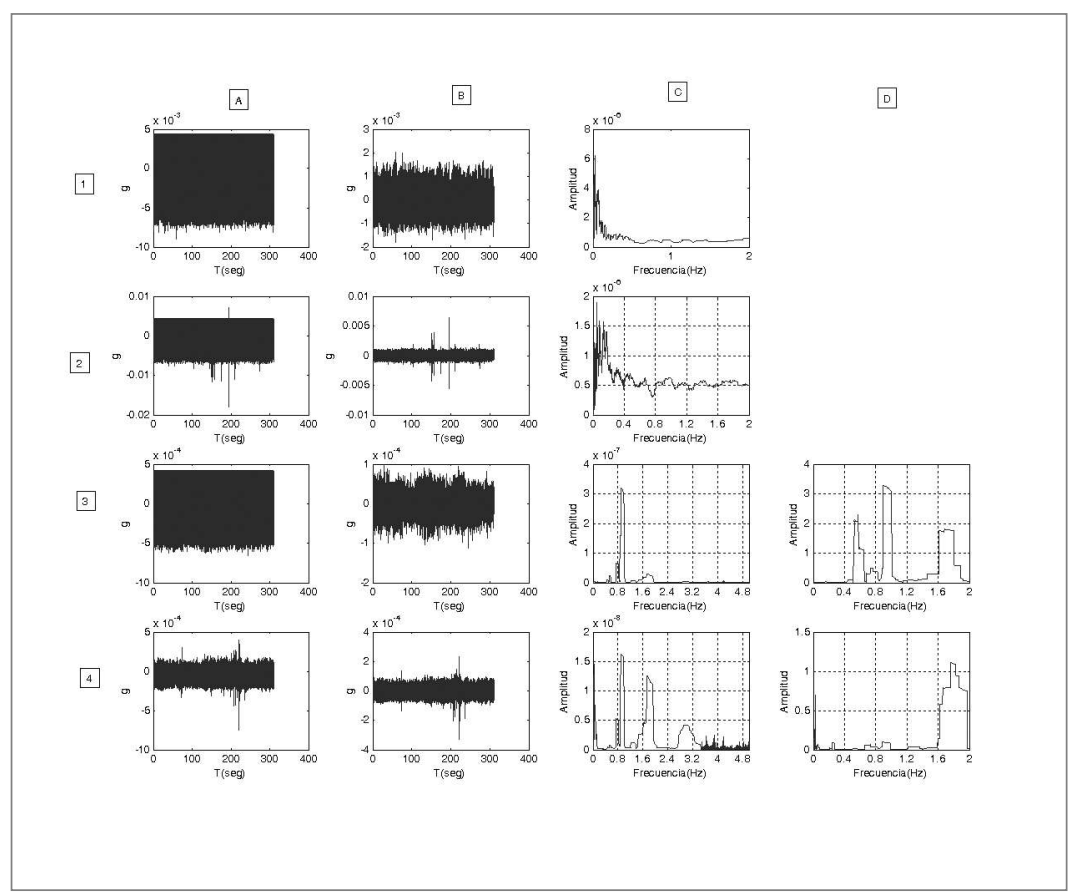

Figura 19. Resultados de vibraciones ambiéntales sentido longitudinal - Febrero 2008 - Sin tráfico³

2 Ibid.

${ }^{3}$ (A1) Acelerograma 1 (sin filtrar). (B1) Acelerograma 1 (filtrado). (C1) Transformada de Fourier del registro del acelerómetro

1 (A2) Acelerograma 2 (sin filtrar). (B2) Acelerograma 2 (filtrado). (C2) Transformada de Fourier del registro del acelerómetro

2.(D2) Función de transferencia C2/C1. (A3) Acelerograma 3 (sin filtrar). (B3) Acelerograma 3 (filtrado). (C3) Transformada 


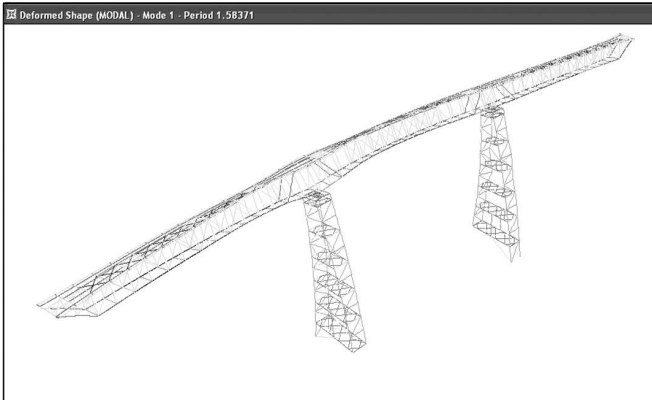

Primer periodo-transversal $(\mathrm{T}=1.58 \mathrm{seg})$

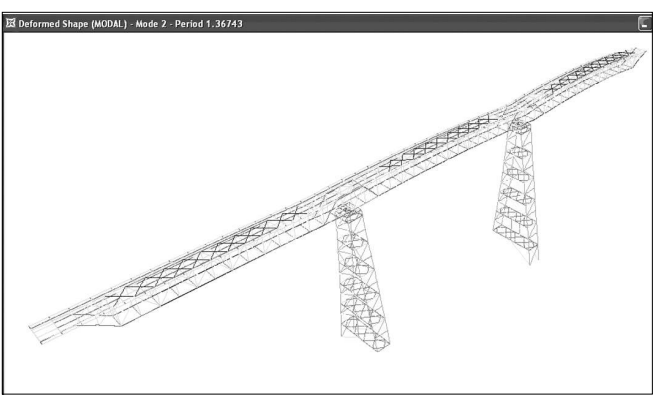

Primer periodo-longitudinal $(\mathrm{T}=1.36 \mathrm{seg})$

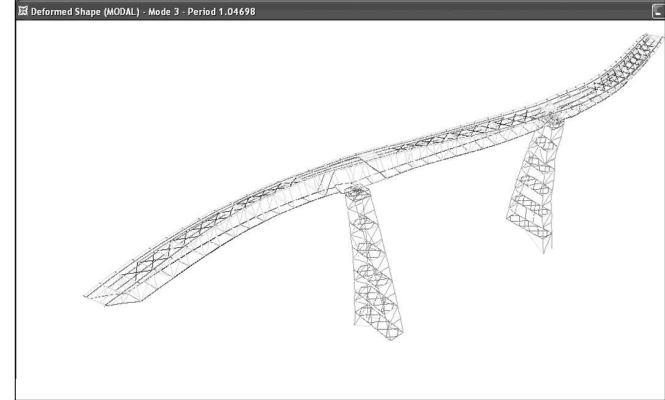

Tercer periodo-torsional ( $\mathrm{T}=1.04 \mathrm{seg})$

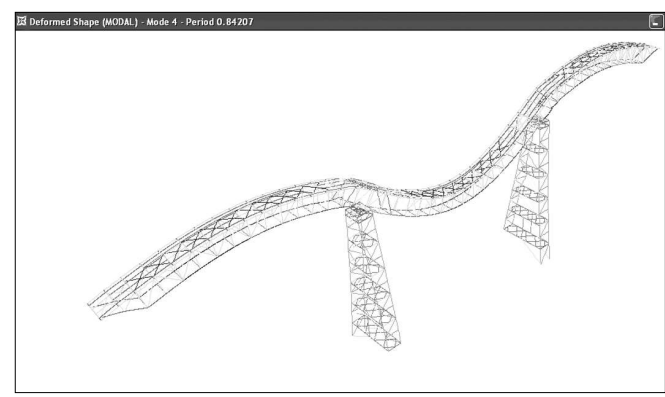

Cuarto periodo - Vertical $(T=0.64 \mathrm{seg})$

Figura 20. Modos de vibración del modelo estructural - losa modelada con parrilla

Tabla 2. Comparación entre los periodos de vibración dominantes del puente en las dos torres del puente basado en la instrumentación de diciembre 2007 y los obtenidos del modelo estructural

(Después del proceso de calibración)

\begin{tabular}{|c|c|c|c|c|c|c|}
\hline & \multicolumn{3}{|c|}{ Torre de Cajamarca } & \multicolumn{3}{|c|}{ Torre de lbague } \\
\hline & \multicolumn{2}{|c|}{ Experimental } & \multirow{2}{*}{$\begin{array}{c}\text { Modelo estructural } \\
\text { Periodo(Seg) }\end{array}$} & \multicolumn{2}{|c|}{ Experimental } & \multirow{2}{*}{\begin{tabular}{|c|} 
Modelo estructural \\
Periodo(Seg) \\
\end{tabular}} \\
\hline & Frecuencia(Hz) & Periodo(Seg) & & Frecuencia(Hz) & Periodo(Seg) & \\
\hline & \multicolumn{3}{|c|}{ Sentido transversal } & \multicolumn{3}{|c|}{ Sentido transversal } \\
\hline 1 & 0.6 & 1.67 & $1.58(5 \%)^{\star}$ & 0.61 & 1.64 & $1.58(4 \%)^{\star}$ \\
\hline 2 & 1.06 & 0.94 & & 0.86 & 1.16 & \\
\hline 3 & 1.43 & 0.70 & & 1.13 & 0.88 & \\
\hline & \multicolumn{3}{|c|}{ Sentido longitudinal } & \multicolumn{3}{|c|}{ Sentido longitudinal } \\
\hline 1 & 0.9 & 1.11 & $1.36(23 \%)^{\star}$ & 0.9 & 1.11 & $1.36(23 \%)^{\star}$ \\
\hline 2 & 1.46 & 0.68 & & 1.5 & 0.67 & \\
\hline 3 & 1.94 & 0.52 & & 1.94 & 0.52 & \\
\hline
\end{tabular}

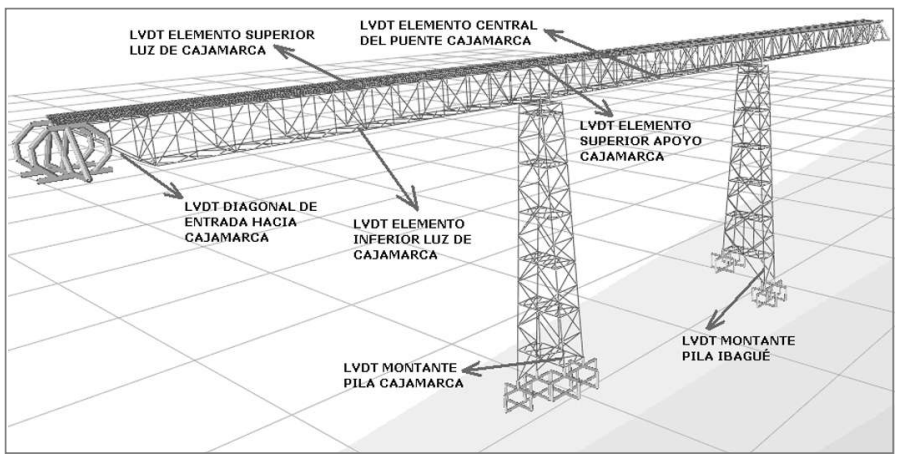

Figura 21. Localización de los sensores 


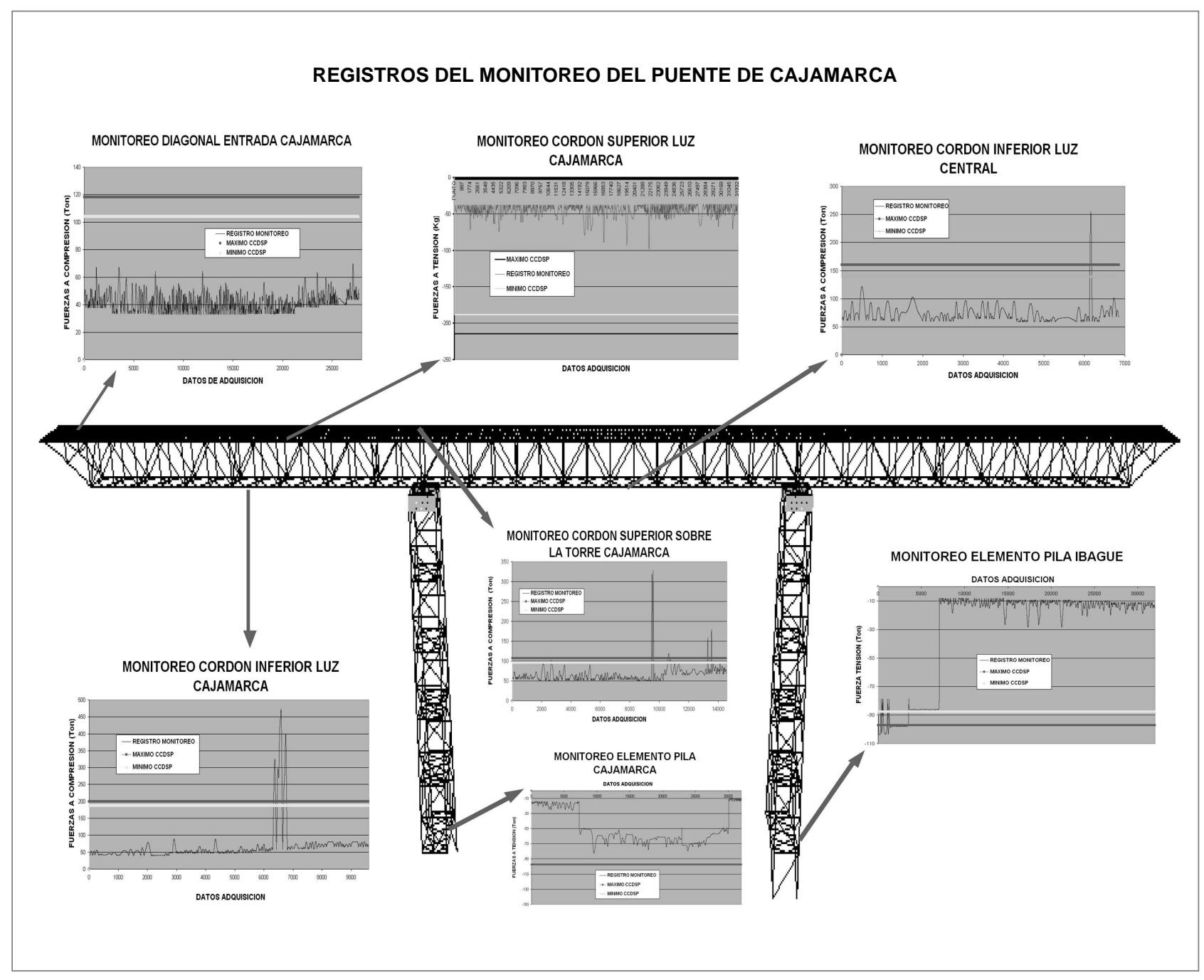

Figura 22. Determinación de picos absolutos de carga del monitoreo del puente vs. Cargas máximas determinados por carga de diseño CCDSP

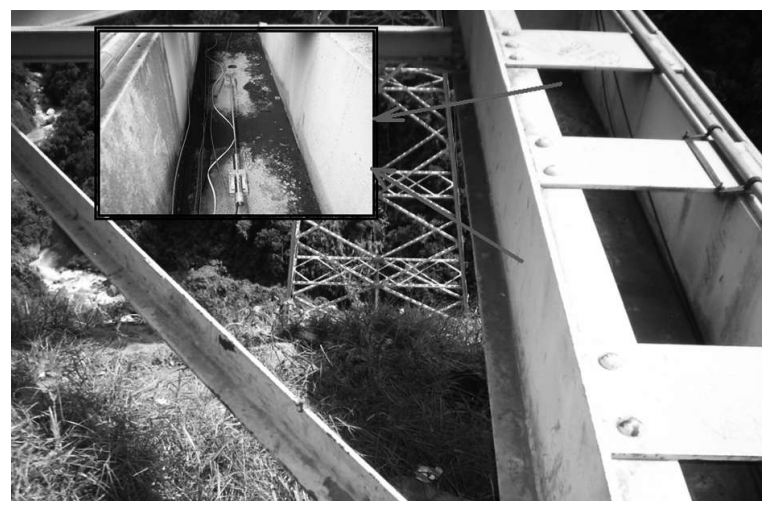

Figura 23. Localización de los dos sensores en el cordón inferior del puente

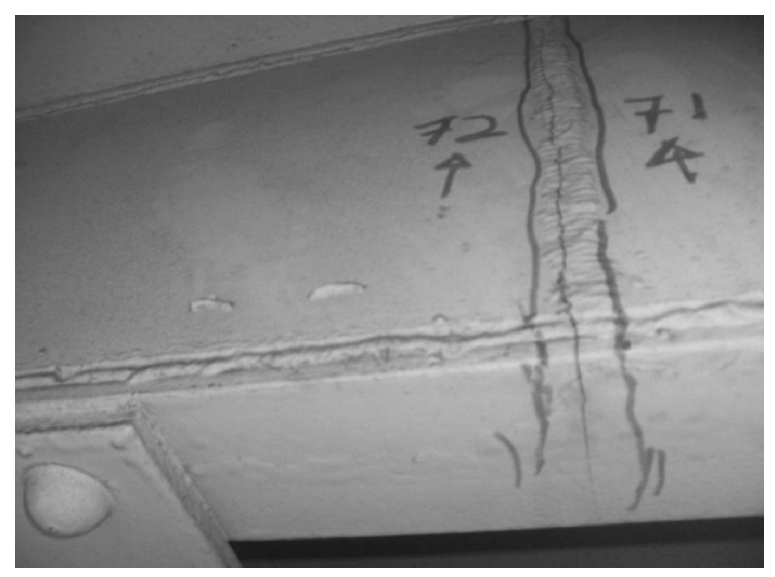

Figura 24. Fisuras en soldaduras que se prolongan hasta platina original 


\section{Análisis de de Resultados}

\subsection{Confiabilidad estructural}

Los valores nominales de factores de seguridad y demás coeficientes estipulados en los códigos son calculados, en su mayoría, mediante técnicas de confiabilidad estructural. Su objetivo es mantener la estructura en un rango de funcionamiento alejado de la falla o con una probabilidad de falla tendiente a cero (Sobrino et al., 1993). Dado que las probabilidades de falla son muy pequeñas (del orden de $10^{-5}$ ) y para facilitar el análisis de los valores hallados, en los códigos se maneja el índice de confiabilidad ( $\beta$ ). Definidos como la inversa de la función normal estándar acumulativa de la probabilidad de falla (Pf):

$$
\beta=\Phi^{-1}\left(P_{f}\right)
$$

La proporcionalidad inversa que existe entre $\beta$ y Pf indican una buena seguridad de la estructura. Para tal efecto se utilizarán las técnicas de confiabilidad que consisten en verificar la probabilidad entre las curvas de la resistencia (R) y de la solicitación (S). Cuando la función $G$ es negativa la estructura se encuentra en condición de falla, cuando es mayor que cero la seguridad de la estructura es aceptable y cuando es igual a cero la estructura se encuentra en condición crítica. Al término $G$ se le conoce como margen de seguridad y se puede expresar como:

$$
G=R-S=0
$$

La distribución de la función límite $(G)$ está determina por esta zona de falla en donde la media de la función $\mathrm{G}\left(\mathrm{m}_{\mathrm{G}}\right)$ es proporcional a la desviación estándar $\left(\mathrm{s}_{\mathrm{G}}\right)$. La proporción de estos dos parámetros es el índice de confiabilidad $(\beta)$, por consiguiente ésta asume una distribución normal que puede evaluarse como:

$$
\begin{gathered}
m_{G}=\beta \cdot s_{G} \\
\beta=\frac{m_{G}}{s_{G}}=\frac{m_{R}-m_{S}}{\sqrt{s_{R}^{2}+s_{S}^{2}}}
\end{gathered}
$$

Donde:

$m_{R}$ : Media de la distribución de la resistencia

$\mathrm{m}_{\mathrm{s}}$ : Media de la distribución de la solicitación

$\mathrm{S}_{\mathrm{R}}$ : Desviación estándar de la distribución de la resistencia

$\mathrm{S}_{\mathrm{S}}$ : D esviación estándar de la distribución de la solicitación

Para el análisis de confiabilidad estructural de este puente, se emplearon las recomendaciones a nivel mundial relacionadas con los rangos de probabilidad de falla permitidos para las estructuras. De acuerdo con lo anterior se revisó el riesgo de esta estructura teniendo en cuenta las siguientes referencias:

- La normativa europea ha sido calibrada para una máxima probabilidad de falla entre $P f=10^{-4}$ y $\mathrm{Pf}=10^{-6}$ (Sobrino J. et al., 1993). Basados en el documento No. 1 Vol. 3 "Traffic loads on bridges" del EUROCODE. Estos rangos de probabilidad de falla en términos de índices de confiabilidad corresponden a: $3.5 \leq \beta \leq 4.5$.

- Los códigos de Estados U nidos y Canadá (AASTHO y O NTARIO) aceptan una probabilidad de falla de 0.001 , lo cual corresponde a un índice de confiabilidad de $\beta=3.09$.

Por otro lado, para el análisis por confiabilidad estructural se seleccionaron las curvas de densidad de probabilidad (normal, logística, Log-normal, valor extremo, etc.), que se ajustan a las funciones de resistencia y solicitación, empleando la técnica de bondad del ajuste para la curva de densidad de probabilidad acumulada de Kolgomorov-Smirnov. Mediante la ecuación 5 se determinó el índice de confiabilidad ( $\beta$ ), el cual se utiliza aún para funciones de distribución de probabilidad diferentes a la normal con resultados satisfactorios. Dicha afirmación se basa en las recomendaciones de algunos autores internacionales (M ays y Tung, 1992 y Ang. A.H S, 1973), los cuales consideran este procedimiento como aproximado con un error mínimo y aceptable. Sin embargo, esta consideración se comprobó analíticamente en el presente artículo, empleando integración numérica y la determinación de la curva resultante G.

Se calculó la probabilidad de falla, la cual corresponde al área bajo de la curva $\mathrm{G}$, que se encuentra entre - $\alpha$ y el punto cero (0), es decir, la zona cuando la curva de solicitación(S) supera a la de resistencia (R). Además se determinó la media y la desviación estándar de la función resultante $G$ y se normalizó. Posteriormente se determinaron el índice de confiabilidad $(\beta)$, que es el inverso del coeficiente de variación del margen de seguridad.

\subsubsection{Para cargas verticales}

Para establecer los índices de confiabilidad por las cargas verticales de los elementos instrumentados, se obtuvieron las curvas de probabilidad de sus solicitaciones producidas por las cargas vivas en el puente. De acuerdo con la teoría de confiabilidad estructural, se puede 
caracterizar la naturaleza variable a partir de la resistencia como de las solicitaciones en términos de las incertidumbres inherentes de cada cual. De esta manera, se determinaron las funciones de probabilidad que se ajustaron matemáticamente de manera óptima a los datos de resistencia calculados con el criterio de ajuste de Kolmogorov-Smirnov. Para las curvas de probabilidad de resistencia se los ensayos mecánicos expresados en el numeral 2.1.

En los elementos principales de las armaduras se encontraron al gunas grietas en las soldaduras que unen el refuerzo solidario con los elementos originales (Figura 24). Esto se halló con las inspecciones visuales realizadas por los contratistas, lo cual ha puesto en duda la confiabilidad y efectividad del refuerzo hecho en 1996. Se presentan a continuación los índices de confiabilidad considerando refuerzos solidarios y no solidarios en los elementos originales (Figura 25 y Figura 26).

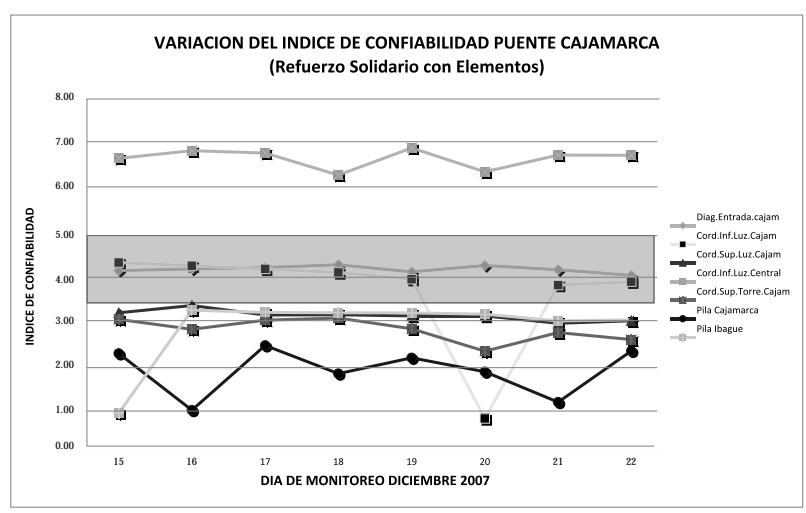

Figura 25. Índices de confiabilidad elementos del Puente Cajamarca considerando que el refuerzo de las secciones transversales es solidario con la sección original

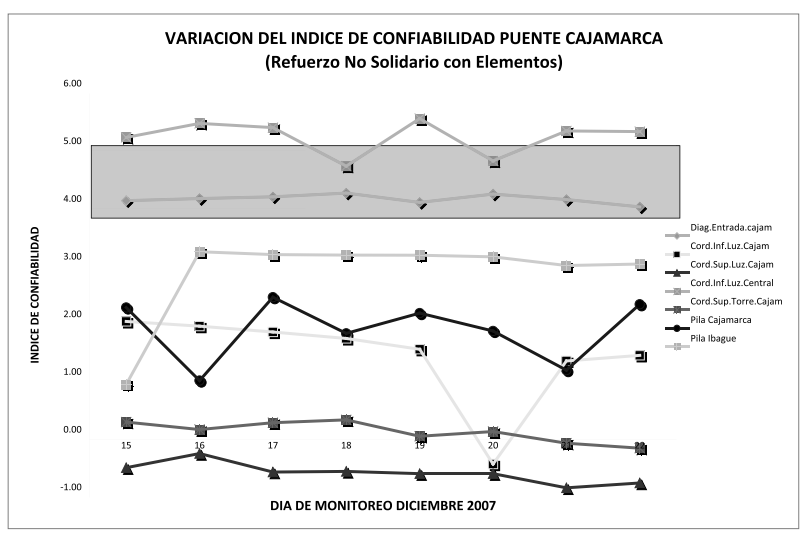

Figura 26. Índices de confiabilidad elementos monitoreados del Puente Cajamarca considerando que el refuerzo de las secciones transversales no es solidario con la sección original
Se deduce que la mayoría de los elementos del puente tienen índices de falla mucho mayores a los recomendados por las normas europeas y americanas. Los mayores índices de falla se encuentran en el cordón superior de la armadura del puente y son preocupantes cuando se considera que el refuerzo sobre el puente no es solidario. Estos resultados desfavorables desde el punto de vista de seguridad del puente, están muy relacionados con las sobrecargas que circulan por el mismo, que pueden generar en cualquier momento su colapso parcial o total (Figura 27 y Figura 28).

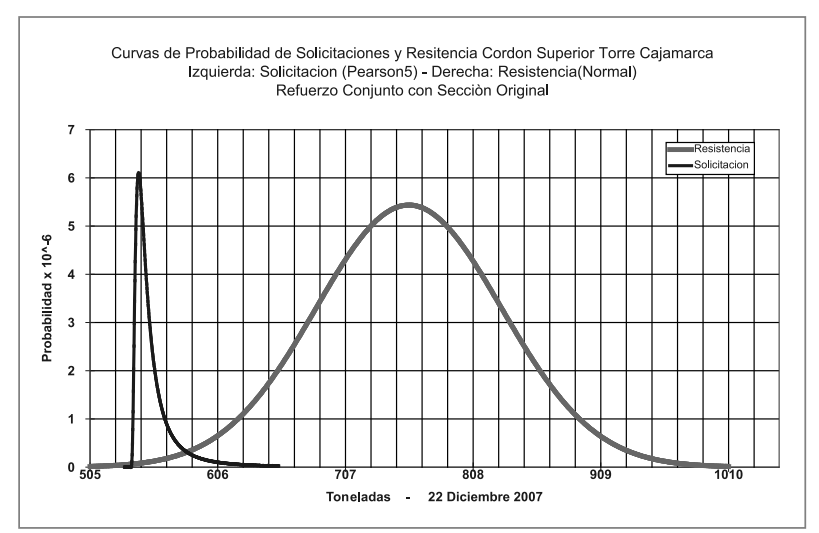

Figura 27. Índice de falla para el cordón superior con refuerzo solidario

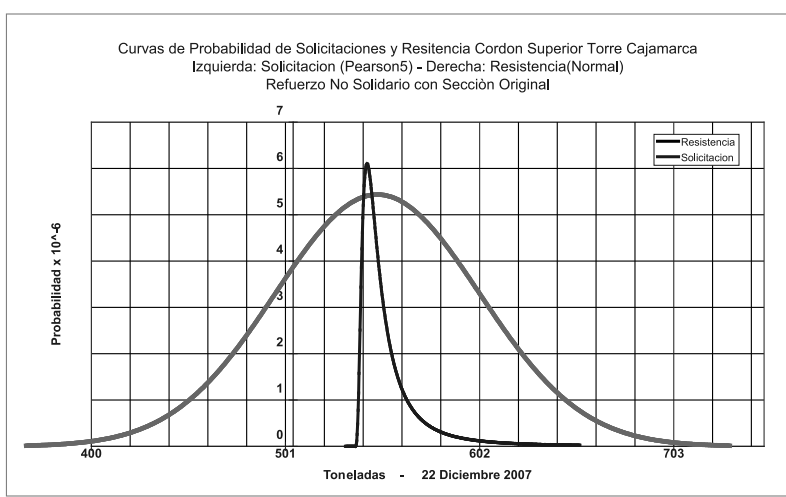

Figura 28. Índice de falla para el cordón superior con refuerzo no solidario

\subsubsection{Para cargas de sismo}

Para poder realizar el análisis de confiabilidad de los elementos del puente desde el punto de vista dinámico, es necesario estimar el factor de modificación de respuesta de dicha estructura, para evaluar en forma aproximada su comportamiento inelástico Para esto se emplearon las recomendaciones de la norma de la referencia (ATC-19), la cual especifica que para estimar este factor con cualquier estructura es necesario evaluar 
la reserva de resistencia de la estructura, su ductilidad y redundancia (Ruíz et al., 2002; Valencia y Valencia, 2008). Con base en lo anterior se estableció que el valor de $\mathrm{R}$ se puede expresar mediante la siguiente ecuación.

$$
R=R_{s} R_{u} R_{r}
$$

En donde:

$\mathrm{R}_{\mathrm{S}}=$ Factor de resistencia

$\mathrm{R}_{\mathrm{u}}=$ Factor de ductilidad

$\mathrm{R}_{\mathrm{R}}=$ Factor de redundancia

Para realizar la evaluación de los factores de la resistencia y la ductilidad de la estructura de este puente se realizó un análisis no lineal estático de "Pushover", empleando las recomendaciones de la norma ATC-40 (Figura 29).

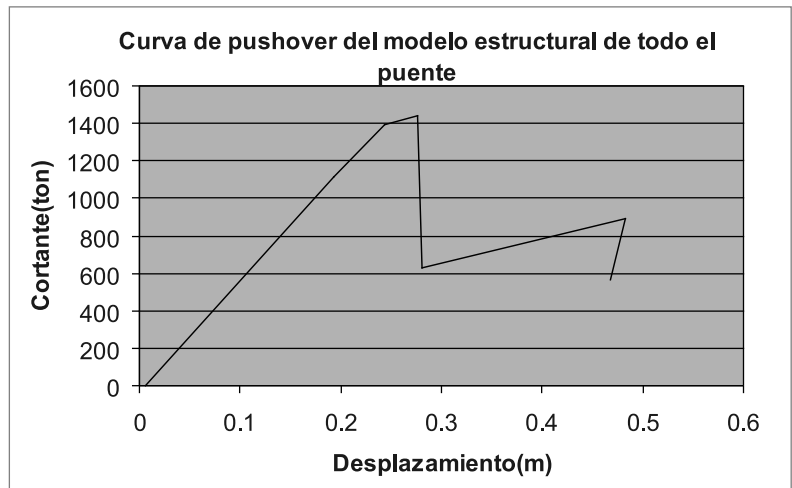

Figura 29. Análisis no lineal de "Pushover", empleando modelo estructural calibrado

El factor de resistencia (Rs) se calculó como el cociente entre el cortante basal máximo alcanzado por el puente $(\mathrm{Vo})$ y el cortante basal de diseño $(\mathrm{Vd})$. Basado en los resultados del "pushover", se encontró que el cortante basal máximo alcanzado por el puente es del orden de 1439.3ton. Para evaluar cortante basal de diseño se realizó un análisis pseudoestático sobre el modelo del puente, teniendo en cuenta que el comportamiento dinámico que se generaría en un sismo es diferente en cada una de las torres. Por lo anterior se decidió emplear el análisis pseudoestático correspondiente al procedimiento de análisis PAS-1 estipulado por el CCDSP, que permite generar cargas sísmicas (aproximadas) diferentes para cada torre. Para este se emplearon los espectros recomendados por el estudio de efectos locales desarrollados en este proyecto. Para el caso del sismo utilizando el espectro de repuesta con un periodo de retorno de 475 años se tiene que el factor de resistencia (Rs) de 1.61.

De acuerdo con la referencia (ATC-19, 1995), el factor de ductilidad está asociado con el desplazamiento máximo adicional al desplazamiento que define el límite elástico de un sistema de un grado de libertad, el cual, generalmente describe su función de rigidez mediante una curva elastoplástica. Para esto se empleó las recomendaciones de (ATC-19, 1995), que sugiere para frecuencias menores $1.0 \mathrm{~Hz}$ (periodos mayores a 1.0 segundos) que el factor Ru es equivalente a la ductilidad. Basados en estas recomendaciones y empleando la curva de Pushover, se encontró que el factor Ru es equivalente 1.13. Este resultado indica que la estructura tiene una baja capacidad de disipación de energía y por lo tanto una ductilidad mínima. El factor de redundancia de esta estructura depende del sistema estructural y su amortiguamiento. Para este puente se consideró un coeficiente de amortiguamiento con respecto al crítico de $2 \%$, por lo cual su factor de redundancia es de 0.8 . Basados con los resultados obtenidos en cada uno de los tres factores, el coeficiente de disipación de energía(R) de esta estructura es de 1.5 (basados en un espectro de un periodo de retorno de 475 años). Como este factor depende del cortante basal de diseño sobre la estructura se tienen diferentes espectros. Este es el cálculo de los factores de disipación para cada una de las acciones sísmicas probables (Tabla 3, Tabla 4 y Figura 30):

Tabla 3. Factores de disipación de energía para diferentes periodos de retorno de los espectros. Caso del $100 \%$ del sismo longitudinal y el $30 \%$ del sismo transversal

\begin{tabular}{|c|c|c|c|c|c|}
\hline \multirow[t]{2}{*}{ Espectros de respuesta } & \multirow[t]{2}{*}{$\begin{array}{l}\text { Redundancia } \\
\text { (Rr) }\end{array}$} & \multicolumn{2}{|c|}{ Resistencia Rs (Vo $=1439.4$ ton) } & \multirow{2}{*}{$\begin{array}{c}\text { Ductilidad (Ru) } \\
\text { Um/Uy }\end{array}$} & \multirow{2}{*}{$\begin{array}{c}\mathbf{R} \\
\mathrm{R}=\mathrm{Rr}^{\star} \mathrm{Rs} \mathrm{s}^{\star} \mathrm{Ru}\end{array}$} \\
\hline & & $\begin{array}{c}\text { Cortante basal máximo } \\
\text { sobre el puente - } \\
(\mathrm{Vd}=\text { Slong+Stra*30\%) }\end{array}$ & $\mathrm{Rs}=\mathrm{Vo} / \mathrm{Vd}$ & & \\
\hline Efectos locales $\operatorname{Tr}=50$ años & 0.8 & 159.14 & 9.04 & 1.13 & 8.2 \\
\hline Efectos locales $\operatorname{Tr}=100$ años & 0.8 & 288.73 & 4.98 & 1.13 & 4.5 \\
\hline Efectos locales $T r=300$ años & 0.8 & 647.71 & 2.22 & 1.13 & 2.0 \\
\hline Efectos locales $T r=475$ años & 0.8 & 893.48 & 1.61 & 1.13 & 1.5 \\
\hline Efectos locales $T r=750$ años & 0.8 & 1300.55 & 1.11 & 1.13 & 1.0 \\
\hline Efectos locales $T r=1000$ años & 0.8 & 1795.82 & 0.80 & 1.13 & 0.7 \\
\hline Efectos locales $T r=2000$ años & 0.8 & 2953.42 & 0.49 & 1.13 & 0.4 \\
\hline Efectos locales $\operatorname{Tr}=3000$ años & 0.8 & 4058.20 & 0.35 & 1.13 & 0.3 \\
\hline Efectos locales $\mathrm{Tr}=5000$ años & 0.8 & 6056.34 & 0.24 & 1.13 & 0.2 \\
\hline
\end{tabular}


Tabla 4. Factores de disipación de energía para diferentes periodos de retorno de los espectros. Caso del $30 \%$ del sismo longitudinal y el $100 \%$ del sismo transversal

\begin{tabular}{|c|c|c|c|c|c|}
\hline \multirow[t]{2}{*}{ Espectros de respuesta } & \multirow[t]{2}{*}{\begin{tabular}{|c|} 
Redundancia( \\
$\mathrm{Rr}$ )
\end{tabular}} & \multicolumn{2}{|c|}{ Resistencia Rs (Vo =1439.4 ton) } & \multirow{2}{*}{$\begin{array}{c}\text { Ductilidad (Ru) } \\
\text { Um/Uy }\end{array}$} & \multirow{2}{*}{$\begin{array}{c}\mathbf{R} \\
\mathrm{R}=\mathrm{Rr}^{*} \mathrm{Rs}^{*} \mathrm{Ru}\end{array}$} \\
\hline & & $\begin{array}{c}\text { Cortante basal máximo } \\
\text { sobre el puente - } \\
(\mathrm{Vd}=\text { Slong+Stra* } 30 \%)\end{array}$ & $\mathrm{Rs}=\mathrm{Vo} / \mathrm{Vd}$ & & \\
\hline Efectos locales $\mathrm{Tr}=50$ años & 0.8 & 135.09 & 10.65 & 1.13 & 9.7 \\
\hline Efectos locales $\mathrm{Tr}=100$ años & 0.8 & 279.86 & 5.14 & 1.13 & 4.7 \\
\hline Efectos locales $\mathrm{Tr}=300$ años & 0.8 & 473.30 & 3.04 & 1.13 & 2.8 \\
\hline Efectos locales $\mathrm{Tr}=475$ años & 0.8 & 668.47 & 2.15 & 1.13 & 2.0 \\
\hline Efectos locales $\mathrm{Tr}=750$ años & 0.8 & 936.46 & 1.54 & 1.13 & 1.4 \\
\hline Efectos locales $\mathrm{Tr}=1000$ años & 0.8 & 1404.59 & 1.02 & 1.13 & 0.9 \\
\hline Efectos locales $T r=2000$ años & 0.8 & 2090.51 & 0.69 & 1.13 & 0.6 \\
\hline Efectos locales $\mathrm{Tr}=3000$ años & 0.8 & 2991.17 & 0.48 & 1.13 & 0.4 \\
\hline Efectos locales $\mathrm{Tr}=5000$ años & 0.8 & 1996.07 & 0.72 & 1.13 & 0.7 \\
\hline
\end{tabular}

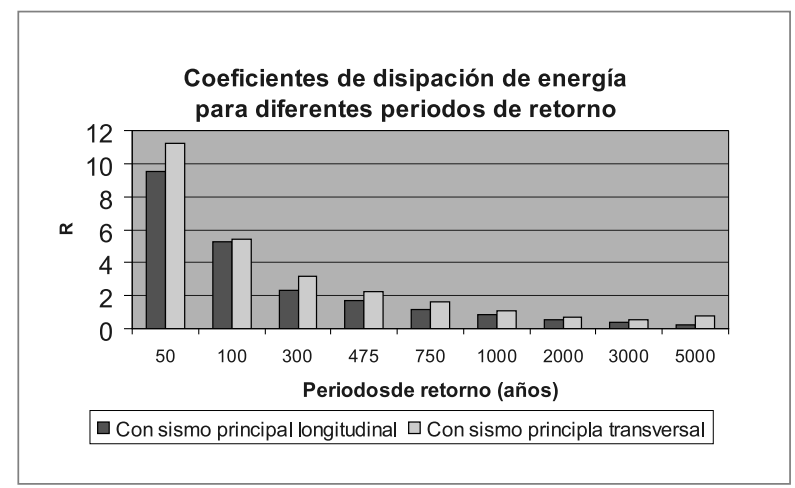

Figura 30. Coeficientes de disipación de energía para cada sismo con diferente periodo de retorno

Para evaluar las curvas de probabilidad de resistencia de cada uno de los elementos se emplearon los resultados de los ensayos a tensión de las muestras de acero del puente. Los elementos principales seleccionados para realizar esta evaluación son los montantes de las torres (en la parte inferior del puente), los cuales están sometidos generalmente a compresión combinada con flexión (en el apoyo) y a tensión para solicitaciones sísmicas altas. También cordones superiores e inferiores de la superestructura (Figura 31). Empleando las recomendaciones del CCDSP, se determinó la resistencia a la compresión de estos elementos, lo cual depende de su limite de fluencia, radio de giro, modulo de elasticidad, longitud efectiva y relación de esbeltez.

Basados en los análisis anteriores y aplicando al modelo estructural calibrado la carga símica producida por cada uno de los espectros de repuesta, con una frecuencia equivalente al inverso del periodo de retorno, se obtuvieron los siguientes índices de confiabilidad para los montantes de las torres. Mediante un análisis seudoestático, se realizó para dos casos de longitud efectiva de dichos elementos ( $K=1.5$ y K=2.0) (Figura 32, 33 y 34).

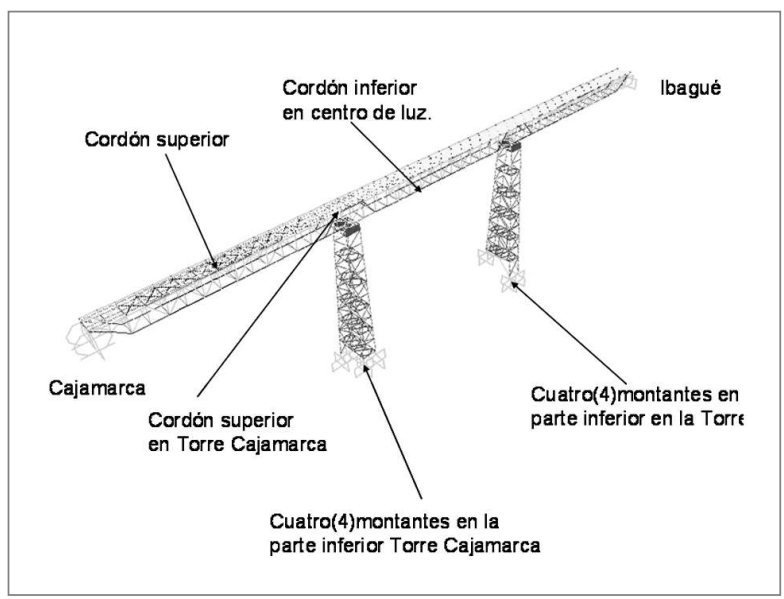

Figura 31. Localización de los elementos seleccionados para la evaluación por confiabilidad estructural

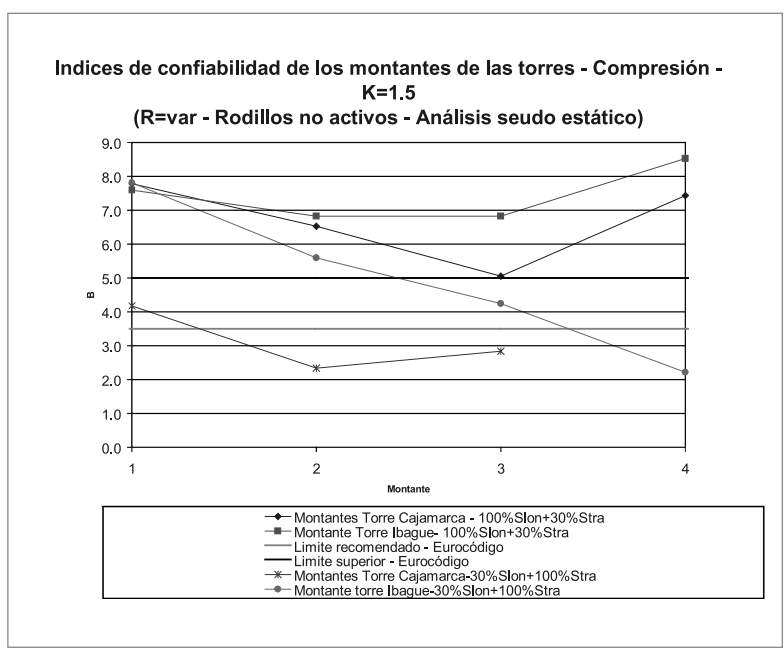

Figura 32. Índices de confiabilidad de montantes para elementos a compresión - $\mathrm{K}=1.5$ 


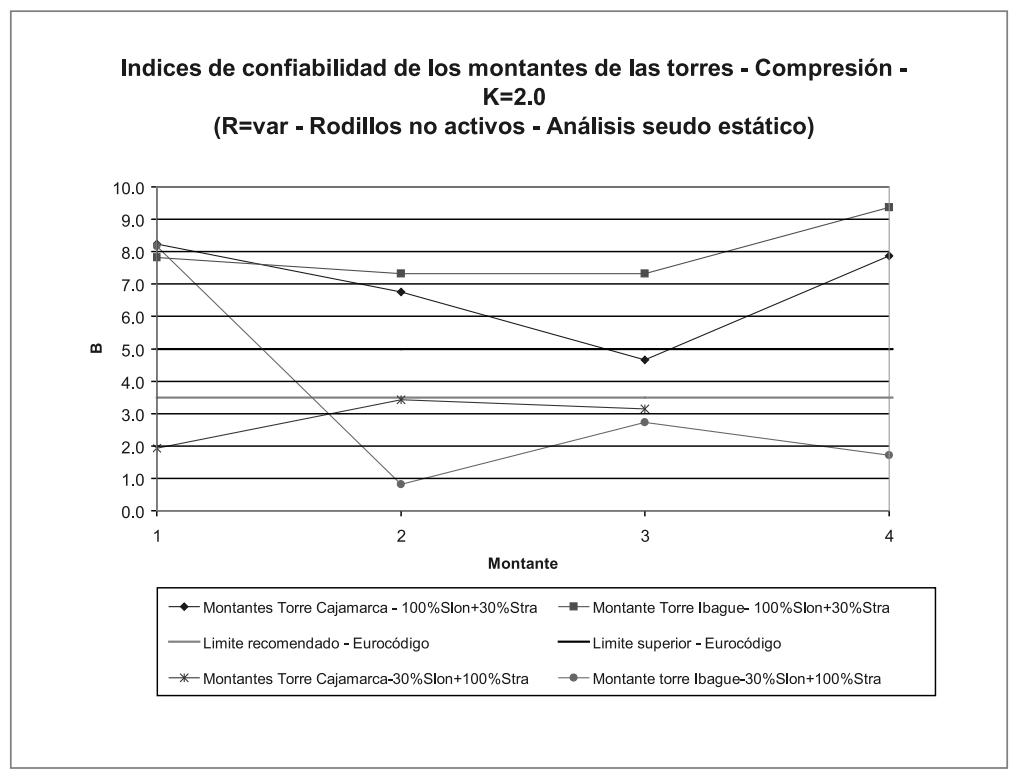

Figura 33. Indices de confiabilidad de montantes para elementos a compresión - K=2.0

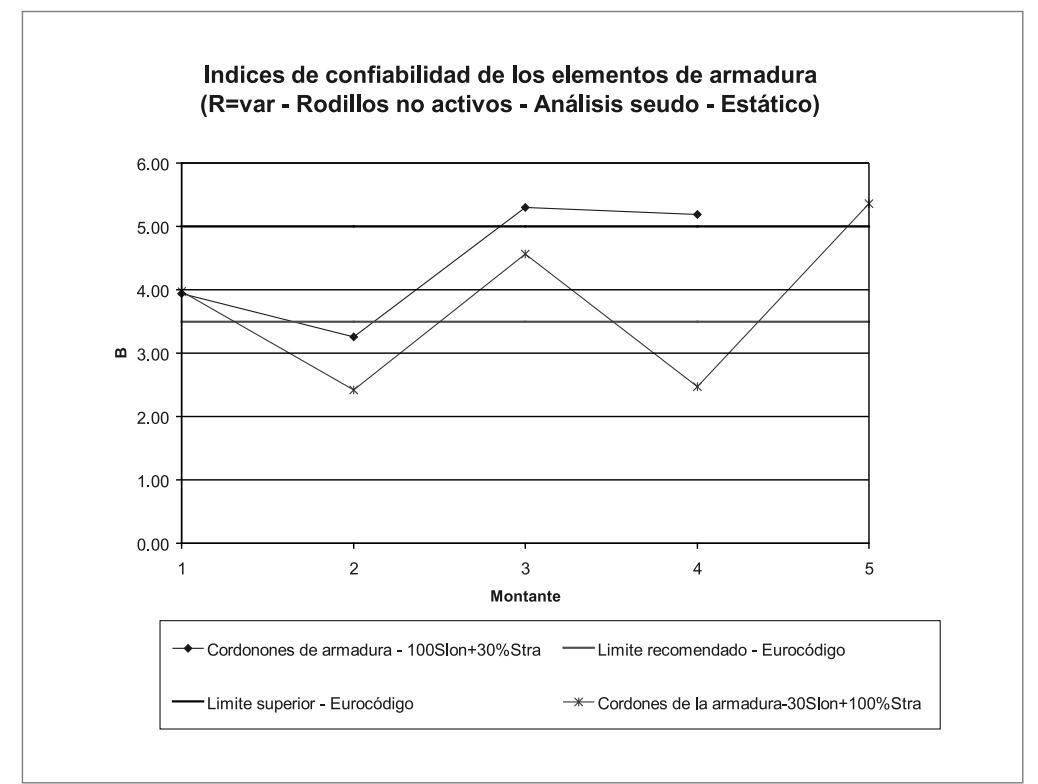

Figura 34. Índices de confiabilidad de los cordones de las armaduras

\section{Conclusiones y recomendaciones}

Las conclusiones y recomendaciones del presente trabajo se transmitieron al INVIAS y a las empresas responsables de la rehabilitación de este puente, convirtiéndose en un insumo complementario en la dinámica de la ingeniería de consulta y de rehabilitación, sirviendo en alguna medida para definir las vulnerabilidades urgentes y prioritarias a solucionar sobre esta estructura. Las conclusiones que se presentan en este trabajo, son específicas para este caso particular de ingeniería, pero pueden servir como referencia para estudios similares relacionados con el estudio de puentes en el mundo. Esto se afirma, porque en el presente trabajo se aprecian las ventajas de emplear análisis de confiabilidad estructural, el cual permite conocer las 
probabilidades de falla, con los cuales se tienen mayor criterio y se pueden tomar decisiones basadas en estudios de riesgo.

También se constituye en otra herramienta, para complementar la evaluación que normalmente hace la ingeniería en Colombia, quienes emplean para estas labores las especificaciones del Código de Diseño (CCDSP), ya que no se tiene una norma o Código para la revisión de puentes existentes. Es importante estos estudios de confiabilidad, porque los factores de mayoración de cargas, los coeficientes de reducción de resistencia y demás especificaciones estipulados en los códigos de diseño, están concebidos para estructuras nuevas y no para la revisión de estructuras existentes, lo cual implica que su empleo puede ser inadecuado, produciéndose resultados que pueden sobredimensionar o sub - dimensionar las labores de rehabilitación.

Del estudio de efectos locales, realizado en el presente estudio, se concluye:

- Q ue el cambio de la topografía y las características del los materiales del lado de Cajamarca con respecto al de Ibagué marcan una amplificación cuatro veces mayor en relación a la aceleración espectral del lado de Ibagué. Se aprecia un efecto local de amplificación sustancial y diferencial del punto $B$ respecto de los otros puntos debido a que se encuentra fundado en los depósitos de cenizas volcánicas los cuales amplifican la respuesta a eventos sísmicos.

- Los resultados de aceleración máxima del terreno en superficie muestran una variación amplia entre 0.2 y $0.5 \mathrm{~g}$, no se aprecian valores significativos de amplificación. Sin embargo, estos valores son altos y pueden ser un factor importante de inestabilidad para las laderas de la margen del Río. Es posible que se presenten fallas de materiales superficiales meteorizados durante un sismo, lo cual es un factor de riesgo para la estabilidad de los apoyos.

- Se sugirió a la Entidad y a los contratistas considerar análisis pseudoestáticos de la estructura en la que se apliquen cargas estáticas equivalentes a las inerciales en la estructura en distintas direcciones, pero diferentes en cada apoyo y compatibles con los rangos de desplazamientos relativos que se presentarán, o considerar el uso de apoyos que permitan el desplazamiento relativo y controlen las cargas en las estructuras como resultado de los movimientos diferenciales del terreno.
Basados en las labores de monitoreo e instrumentación, se encontró:

- Sobrecargas importantes que afecta la seguridad estructural principalmente en elementos del puente tales como: cordón superior de las armaduras, montantes de las torres, apoyos y conexiones remachadas y soldadas. Este nivel de sobrecarga combinada con los daños y defectos estructurales detectados (grietas en soldaduras y secciones principales), son un riesgo inadmisible para la estabilidad de la estructura. Para esto se sugirió a la Entidad complementar las políticas del Estado sobre el control de cargas en puentes y pavimentos en la red Vial Nacional, para no afectar la estabilidad de las obras de infraestructura vial y evitar un gasto innecesario.

- Se encontró que la rehabilitación estructura realizada para este puente en 1996, no tiene la efectividad deseada, lo cual representa para futuros trabajos revaluar este tipo refuerzo.

Basados en el estudio de confiabilidad estructural, se detectó:

- Que cinco de los siete elementos principales monitoreados, tienen para los efectos de cargas verticales, altas probabilidades de falla, mayores a las estipuladas por las normas europeas y americana. Estas probabilidades de falla fueron más evidentes cuando se identificó que las platinas de refuerzo colocadas en el puente en 1996 no son completamente solidarias con las secciones originarias del mismo. En conclusión, varios elementos no cumplen con los requisitos de seguridad básicos, por lo cual son vulnerables y su rehabilitación es prioritaria.

- Desde el punto de vista sísmico, que los montantes de las torres de este puente tienen probabilidades de falla mayores a los recomendados por las normas internacionles. Para hallar estos resultados se emplearon los resultados del estudio de efectos locales del sitio; el modelo estructural calibrado y los ensayos mecánicos de ocho muestras de acero. De esta forma se concluye que la vulnerabilidad sísmica de este puente es muy alta y se requiere ejecutar urgentemente obras de rehabilitación, que permitan mejorar su comportamiento dinámico desde el punto de vista de rigidez, ductilidad y resistencia. 
A nivel de recomendaciones, se le sugirió a los funcionarios del IN VIAS y a las empresas de ingeniera, lo siguiente:

- Se deben revisar los apoyos de la superestructura en los estribos y las torres, para evaluar el comportamiento y vulnerabilidad en caso de un evento sísmico.

- Revisar las conexiones remachadas y soldadas empleando ensayos no destructivos para verificar el estado estructural de cada una de las platinas y remaches.

- Evaluar que los refuerzos sobre el puente no se hagan únicamente con soldadura sino mediante pernos de alta resistencia u otro sistema. Buscando que las labores de rehabilitación sean más eficientes y de mayor calidad.

- Revisar los anclajes de las torres en los cajones de concreto, su capacidad y comportamiento ante un evento sísmico.

- Realizar un estudio de estabilidad de las laderas, ya que pueden presentarse vulnerabilidades que afecten la estabilidad de las torres.

- Verificar la estabilidad y capacidad de los dos estribos pues no hay información sobre el tipo de cimentación y vulnerabilidad ante un evento sísmico.

También que es importante monitorear esta estructura después de su rehabilitación para verificar la efectividad de las obras y el nivel de seguridad, empleando técnicas de confiabilidad estructural. Además realizar un estudio especializado de fatiga, que logre determinar la vida remanente de las conexiones y elementos de esta estructura.Finalmente este estudio fue seleccionado dentro de los programas "Exitosos" en la Conferencia Regional de Educación Superior(2008), por los resultados logrados, que son tecnológicos e innovadores, a través de la alianza Empresa- U niversidad y Estado.

\section{Agradecimientos}

Los autores expresan su agradecimiento al Instituto Nacional de Vías, por el apoyo logístico y económico para el desarrollo de este proyecto. Especialmente a los ingenieros Virginia Ramos y Libardo Santacruz. También a las empresas de ingenierías, por toda la información que se nos suministró y el trabajo en equipo que se desarrolló. También el Ingeniero Daniel Ruiz, por las referencias suministradas para el análisis sísmico de este puente. Igualmente al Ingeniero Jorge Alonso Prieto quien nos presto y autorizo el uso de su programa para la generación de espectros.

\section{Referencias}

Ambrasseys N. N ., Simpson K. A. y Bommer J. J. (1996), Prediction of horizontal response spectra in Europe. Earthquake engineering and Structural Dynamics, Vol. 25, pp 371-446.

Ang A H-S. (1973), "Structural Risk Analysis and ReliabilityBased Design", Journal of Structural Engineering Division, ASCE, Vol 99, no. ST9.

Asociación de Ingeniería Sísmica (AIS). (1995), Código Colombiano de Diseño Sísmico de Puentes. Asociación de Ingeniería Sísmica (AIS). (1996), Estudio general de amenaza sísmica de Colombia. ATC-19 (Applied Technology Council). (1995), Structural response modification factors.

Binaria Ltda. (2006), “Estudio de vibraciones ambientales del puente de la Avenida calle 170 por autopista Norte", Trabajo de consultoría desarrollado para el Instituto de Desarrollo U rbano (IDU-Bogotá).

Gallego M. (2007), "Evaluación dinámica de dos(2) puentes peatonales de Bogotá", Trabajo para el Instituto de Desarrollo U rbano(IDU - Bogotá), Binaria Ltda.

IN GEO MINAS-U NIANDES (1996), Estudio general de amenaza sísmica para Colombia. Bogotá

Johnson R. A. (1973), An earthquake spectrum prediction technique. Bulletin of the Seismological Society of America, Vol 63, pp 1255-1274.

M ays L. y Tung Y. (1992), "Hydrosystems engineering \& Management", McG raw -Hill Series in Water Resources and environmental engineering.

McGuire R. K. (1977), Seismic design spectra and mapping procedures using hazard analysis based directly on oscillator response. Earthquake engineering and structural dynamics, Vol. 5, pp 211234.

Muñoz E. (2002), "Estudio de las causas del colapso de algunos puentes en Colombia". Ingeniería y U niversidad, Vol. 6, No1, pp 33-48.

Muñoz E., Daza R. y Salazar F. (2002), “M etodología de evaluación estructural de puentes metálicos por técnicas de fiabilidad estructural". Revista Ingeniería de Construcción, Vol 17, №1, pp 44-52.

Muñoz E. y Valbuena E. (2005), "Evaluación de un puente en acero mediante criterios de confiabilidad parcial", Revista de la Asociación Técnica de Carreteras, №110, 
pp 49-60.

Muñoz E., N uñez F., Rodríguez W., O talora C. y Ruiz D. (2006), "Confiabilidad estructural apoyada en monitoreo e instrumentación: Aplicación en un puente de acero", Revista Ingeniería de Construcción, Vol 21, №2, pp 87-100.

National O ceanic \& Atmospheric Administration (NOAA), SMCAT (Strong Motion Catalog). (1996).

Newmark N. M. y Hall W. J. (1982), Earthquake spectra and design. EERI monograph, Berkeley, California.

Ordaz M. y Montoya C. (2002), Programa DEG TRA 2000, Instituto de Ingeniería. Universidad Autónoma de México.

Ruiz D.M., y Sarria Molina A. (2002), Efecto de las diferentes componentes sísmicas de movimiento sobre las naves industriales de grandes luces. Revista de Ingeniería. Bogotá. N o. 15, pp 46-55.

SAP (2000), Structural Análisis Program, Computers and Structures Inc, U niversity Av. Berkeley C.A. Sarrazin M ., M oroni M. O, Q uintana R. y Soto P. (2002), "Respuesta Sísmica de Puentes Chilenos con Apoyos Aislantes", Revista Internacional de D esastres $N$ aturales, Accidentes e Infraestructura Civil, Vol 2, №2, pp 31-48.

Sobrino J.A., y Casas R. (1993), M etodología de Evaluación Estructural de Puentes Existentes: Aplicación a un caso Real. En: Hormigón y Acero. Barcelona. UPC. IV Trimestre, pp 107-124.

Tanner P. y Sobrino J. A. (1998a), ¿Cuánta Seguridad Necesitan las Estructuras? Calibración de Códigos. En: Curso de Estudios Mayores de la Construcción. (1998: Barcelona). Fiabilidad Estructural. Tratamiento de las Acciones Especiales, pp 19.

Tanner P. y Sobrino J. A. (1998b), Acciones en Puentes. En: Curso de Estudios Mayores de la Construcción.. Fiabilidad Estructural. Tratamiento de las Acciones Especiales, pp 12.

Valencia G. C., y Valencia D. R. (2008), “Evaluación del coeficiente de disipación de energía, $R$, para algunos tipos de estructuras de acero" Ingeniería e Investigación, U niversidad Nacional de Colombia, No 1, pp 41-49.

Wells D. y Compersmith K. (1994), New empirical relationships among magnitude, rupture width, rupture area and surface displacement. Bulletin of the Seismological Society of America, Vol. 84, pp 974-1003. 